\title{
A low dose of beryllium exposure influences gut microbiota and promotes metabolic syndrome.
}

\section{Kwang Hyun Cha}

Korea Institute of Science and Technology https://orcid.org/0000-0003-3001-3067

Jung-Seok Yang

Korea Institute of Science and Technology

Dae-Geun Song

Korea Institute of Science and Technology

Kye-Yoon Yoon

Korea Institute of Science and Technology

Erdenedolgor Erdene-Ochir

Korea Institute of Science and Technology College of Business

Kyung-A Kim

Korea Institute of Science and Technology

Hyo Shin Yoon

Seoul National University School of Public health

Hyun Ju You

Seoul National University School of Public Health

Mi Young Lim

Korea Food Research Institute

Boram Seo

Seoul National University School of Public Health

Jong Beom Jin

Korea Institute of Science and Technology

Cheol-Ho Pan

Korea Institute of Science and Technology

GwangPyo Ko ( $\nabla$ gko@snu.ac.kr)

https://orcid.org/0000-0002-0524-2194

Research

Keywords: metabolic disorder, fecal elements, gut microbiota, beryllium exposure

Posted Date: March 11th, 2020 
DOI: https://doi.org/10.21203/rs.3.rs-16928/v1

License: (a) (i) This work is licensed under a Creative Commons Attribution 4.0 International License. Read Full License 


\section{Abstract}

\section{Background}

Elements, including essential minerals and metals, play an important role in human biological functions. Some studies have suggested that exposure to certain elements such as arsenic and cadmium can disturb gut microbiota and result in metabolic disorders. Despite considerable evidence that exposure to toxic heavy metals is significantly associated with host metabolic disorder, few studies have investigated the relationships between fecal elements, gut microbiota, and host metabolism. In order to gain a deeper understanding of the impact of various fecal elements on gut microbiota and metabolic disorder, we have to investigate fecal elements from human stools for association study and demonstrate that certain elements have the causal effect on metabolic disease and changes in gut microbiota.

Results

We analyzed 28 elements from 304 feces of human twins and evaluated the effects of fecal elements on both gut microbiota and metabolic disorder. Fecal beryllium content was found significantly correlated with biomarkers of metabolic disease and metabolic disease-related gut microbiota such as Akkermansia and Bifidobacterium . In vitro human fecal culture showed marked reduction of species evenness and Bifidobacterium abundance after beryllium treatment. Notably, in mice fed a high-fat diet, $30 \mathrm{ppb}$ of beryllium exposure resulted in significant body weight gain and increased plasma biomarkers for metabolic disorder with an altered microbial community. Beryllium exposure also affected cecal short chain fatty acid profiles, colonic function, and inflammation.

\section{Conclusions}

Our findings indicate that low doses of beryllium, almost similar to the current criteria for beryllium in drinking water, perturb the gut microbiota and worsen metabolic disorders, which is significant implication in public health.

\section{Background}

Chronic metabolic syndrome (MetS), such as obesity and diabetes, has become increasingly prevalent worldwide. This increasing trend within the last 30 years is believed to be caused by excessive caloric intake and lack of physical activity [1]. However, there is considerable evidence that other risk factors such as exposure to environmental pollutants and chemicals may be involved in MetS etiologies [2]. Recent studies indicate that the effects of dietary intake on the gut microbiome can surpass the genetic factors and immune responses of individuals, emphasizing a crucial role for external factors such as antibiotics, drugs, and environmental chemicals [3]. Chemicals that promote MetS have been functionally defined as obesogens and diabetogens because they can affect lipid accumulation and adipogenesis as well as induce a shift in energy balance, change basal metabolic rates, and alter hormonal or neuronal control. 
Elements, including essential minerals and metals, play an important role in human biological functions [4]. A deficiency in essential minerals or an excess of toxic trace elements have been linked to chronic MetS. It has been reported that calcium or zinc supplements can reduce body weight and improve host adiposity [5,6]. On the other hand, exposure to arsenic, cadmium, and lead has been associated with diabetes, cardiovascular disease, hypertension, and peripheral vascular disease [7, 8]. In particular, some studies have suggested that exposure to certain elements such as arsenic and cadmium can disturb gut microbiota and result in metabolic disorders [9-11], indicating that the obesogenic effects of heavy metals may be associated with gut microbiota.

Recent studies have also suggested a possible role for gut microbiota in MetS, demonstrating how microbial alteration affects energy intake and induces low-grade inflammation by metabolic endotoxemia $[12,13]$. Given that the close link between MetS and gut microbiota has recently been highlighted and that most elements are excreted in feces rather than absorbed into the host via the digestive tract, it is important to elucidate the relationship between various elements with the human gut microbiota and MetS status. However, most studies on elements and MetS were performed on the host side without considering the role of gut microbiota and have examined a few elements, such as arsenic and cadmium. Moreover, some approaches that considered the link between elements and gut microbiota were mostly performed on animals only, which has limited translational implication to human.

Here, we demonstrated how various fecal elements influence on both MetS and gut microbiota using 304 human twins adjusted for human genetic factors and animal models. For beryllium (Be), which was significantly correlated with biomarkers of MetS and MetS-related gut microbiota, we assessed whether a low dose of Be exposure would have a causal relationship with gut microbial changes and MetS deterioration. A schematic diagram of the experimental study design was shown in Additional file 1: Figure S1.

\section{Results}

Association between fecal elements and clinical MetS indices.

We measured the content of 28 elements in human feces and divided them into two groups, the major fecal elements group ( $\mathrm{Ca}, \mathrm{P}, \mathrm{K}, \mathrm{Mg}, \mathrm{S}$, and $\mathrm{Na}$ ) and the trace elements group ( $\mathrm{Fe}, \mathrm{Zn}, \mathrm{Al}, \mathrm{Mn}, \mathrm{Sr}, \mathrm{Cu}, \mathrm{Ti}, \mathrm{Ba}$, $\mathrm{Rb}, \mathrm{Cr}, \mathrm{Ga}, \mathrm{As}, \mathrm{Se}, \mathrm{Cd}, \mathrm{Co}, \mathrm{V}, \mathrm{Pb}, \mathrm{U}, \mathrm{Li}, \mathrm{Cs}, \mathrm{Bi}$, and $\mathrm{Be}$ ) according to their relative abundance (1\%; Additional file 2: Table S1). Ca was the most abundant fecal element in the healthy group, whereas $\mathrm{K}$ was the highest in the MetS group. The total concentration of elements was higher in the healthy group ( $13,947 \mu \mathrm{g} / \mathrm{g})$ than in the MetS group $(\sim 11,381 \mu \mathrm{g} / \mathrm{g})$. Moreover, the average relative abundance values of $\mathrm{K}, \mathrm{Mg}, \mathrm{S}, \mathrm{Na}, \mathrm{Al}, \mathrm{Ti}, \mathrm{Rb}, \mathrm{Cr}, \mathrm{As}, \mathrm{Se}, \mathrm{Cd}, \mathrm{Co}, \mathrm{Pb}$, and $\mathrm{Be}$ were higher in the MetS group than in the healthy group. To investigate the fecal elements associated with MetS status, we analyzed the correlation between 28 fecal elements and each MetS-related index, which includes high-density lipoprotein cholesterol (HDL), total cholesterol (tCholesterol), low-density lipoprotein cholesterol (LDL), body mass index (BMI), triglyceride, uric acid, aspartate transaminase (AST), alanine transaminase (ALT), waist 
circumference (Waist), fasting blood sugar (FBS), systolic and diastolic blood pressure (SBP and DBP), high sensitivity C-reactive protein (hsCRP), and fasting blood insulin (FBInsulin), using Spearman's rank correlation test (Fig. 1a). When fecal elements were sorted based on their correlation with MetS, two elements, $\mathrm{Be}$ and $\mathrm{Ca}$, showed the strongest correlation compared with that of other elements. Single MetS-related biomarkers such as BMI, AST, ALT, Waist, hsCRP, and FBInsulin showed a significant positive correlation with Be. In contrast, Ca showed a significant negative correlation with BMI, Waist, FBS, and SBP biomarkers.

Association between fecal elements and human gut microbial populations.

We next examined whether certain elements can affect the a-diversity of the microbial community such as Chao1 richness and the Simpson and Shannon indices (Additional file 3: Figure S2). When diversity analysis was carried out based on the tertile levels of each element, Be showed a significant reduction in microbial diversity. Simpson and Shannon diversity indices, which indicate species evenness, were significantly decreased in the high tertile level of Be. To assess the overall correlation between fecal elements and gut microbiota, we filtered microbial taxa by excluding low-abundance taxa $(<0.1 \%$ of mean relative abundance) and calculated Spearman's rank correlation coefficient between 28 fecal elements and 29 gut microbiota genera (Fig. 1b). Some elements were grouped in a separate cluster, indicating that there may be a robust link between certain fecal elements and the shaping of the gut microbiota community. In particular, the four genera (Akkermansia, Bifidobacterium, Odoribacter, and Parabacteroides) that showed a significant abundance in the healthy group in our previous study [14] were positively associated with $\mathrm{Ca}$. Moreover, Be had a strong positive correlation with Sutterella, the most significantly increased genera from the MetS group in our study, and a robust negative correlation with both Akkermansia and Bifidobacterium, which were reported to attenuate MetS $[15,16]$. To characterize the association by multivariate analysis adjusting for potential confounding factors, MaAsLin analysis was performed for fecal Be and $\mathrm{Ca}$ with adjustments for age, sex, smoking status, monozygotic (MZ) and dizygotic (DZ) twin, and family relationship (Additional file 4: Table S2). The results indicated that MetS-related gut microbial genera such as Bifidobacterium and Akkermansia were still significantly associated with Be and $\mathrm{Ca}$ after the adjustment.

Microbial functional analysis based on fecal Be status.

We acquired high-resolution ${ }^{1} \mathrm{H}-\mathrm{NMR}$ spectra, and 67 metabolites were assigned and quantified for metabolomic analysis. These metabolites were analyzed using supervised multivariate analysis (PLSDA). We observed differences in the score plots between the low and high tertile Be groups (Fig. 2a). According to the corresponding loading plot, 12 metabolites were identified as discriminating metabolites (VIP > 1; Fig. 2b). Fumarate, L-tyrosine, glycine, putrescine, L-lysine, L-isoleucine, glyceraldehyde, and cadaverine levels were found proportionally decreased to those of $\mathrm{Be}$, whereas trimethylamine, acetate, Ltryptophan, and heptanoate increased. To evaluate the alteration in gut microbial functions based on fecal Be status, we used PICRUSt to infer functional metagenomes from their reference genomes based on 16S rRNA gene sequences. Functional pathways that were differentially abundant between the low 
and high tertile Be groups were identified and represented as histograms of the linear discriminant analysis (LDA) scores (Fig. 2c). The oxidative phosphorylation pathway was overrepresented in the highBe group, suggesting that Be was likely to be associated with oxidative chemical reactions in the gut environment. We also observed that energy metabolism, such as methane, pyruvate, arginine, and proline metabolism, was enriched in the low-Be group compared with the high group. Thus, we found that $\mathrm{Be}$ status in the gut may influence not only microbial composition but also subsequent metabolic function of gut microbiota.

Be induces gut microbiome changes in in vitro cultured human feces.

We next carried out in vitro anaerobic colonic culture using human feces to analyze the impact of Be exposure on the human microbiome and to evaluate the direct relationship between Be and the gut microbiota while excluding the host effect. After $24 \mathrm{~h}$ of in vitro cultivation, the microbial composition remained identical to the original human fecal samples except for a slight reduction in Bacteroides (Fig. 3a). Notably, Be treatment markedly altered microbial composition. The Be treatment groups were easily distinguished from the Be untreated group, suggesting that exposure to Be directly alters the microbial community (Fig. 3b). In particular, there was a significant reduction in Coprococcus, Bifidobacterium, Ruminococcus, and Roseburia, whereas Bilophila significantly increased in the Be exposure groups compared with the control group (Fig. 3c).

Be promotes MetS in high-fat diet (HFD)-fed mice.

To determine the influence of Be exposure on host metabolism and the microbial community, we performed in vivo mouse experiments. We first examined body weight gain (\%) and feed intake over 7 weeks. In the normal diet (ND) group, there was no significant increase in body weight gain after $30 \mathrm{ppb}$ of Be exposure and feed intake remained constant (Fig. 4a). However, in the HFD group, $30 \mathrm{ppb}$ of Be exposure resulted in modest but significant gains in overall weight, indicating that Be may accelerate HFD-induced obesity (Fig. 3b). Although 3 ppb of Be did not lead to a significant weight gain over 7 weeks, the weight gain was similar to that of the weight gain observed within the first 2 weeks after treatment with $30 \mathrm{ppb}$ Be. Such an increase in body weight gain was linked to the upsurge of feed consumption during early development. There was no significant difference in the intake of water between all groups, regardless of ND or HFD (Additional file 5: Figure S3). The theoretical average Be exposure was $43.84 \mathrm{ng} /$ week, $300.50 \mathrm{ng} /$ week, and $337.47 \mathrm{ng} /$ week in HFD-Be3, ND-Be30, and HFD-Be30 groups, respectively. Next, we investigated the possible causal relationship between Be exposure and host adiposity. In the ND group, MetS-related biomarkers such as plasma concentrations of triglycerides (TGs), glucose, insulin, leptin, and adiponectin did not show any significant differences after Be exposure, whereas epididymal white adipose tissue (eWAT) weight was significantly higher than in control after exposure to $30 \mathrm{ppb}$ Be (Additional file 6: Figure S4). For the HFD group however and similar to the results of body weight gain, Be exposure worsened host adiposity (Fig. 4c). Plasma levels of TGs, glucose, and insulin were also markedly increased in HFD-Be30. The adipokine leptin also increased after $30 \mathrm{ppb} \mathrm{Be}$ exposure, whereas adiponectin significantly decreased in both HFD-Be3 and HFD-Be30 groups compared 
with control. We were unable to observe a significant difference in eWAT weight across the three HFD groups, possibly because the eWAT was almost saturated due to the 7-week HFD. Taken together, these findings indicate that long-term exposure to Be may promote metabolic disorders in HFD mice.

Be alters the composition of gut microbiota in HFD mice.

To determine whether the metabolic disorder developed after Be exposure is associated with changes in microbial composition, we analyzed the cecal microbiome. In $\beta$-diversity analysis, HFD-Be3 and HFDBe30 showed marked microbial community changes compared with that of the control group, and this clear microbial shift pattern was not seen in ND groups (Fig. 5a and Additional file 7: Figure S5). To further examine the changes in the gut microbial community of the HFD group due to Be exposure, we performed fecal microbiome analysis using mice fecal samples collected over 5 weeks (Fig. 5b). Starting at week 0 week, prior to Be treatment, three HFD groups were gathered in one cluster. However, by week 5 , the Be exposure groups moved to another cluster. Remarkably, these changes in microbial clusters occurred within a week. We investigated a-diversity, such as Chao1 richness and the Simpson and Shannon diversity indices, to examine whether the reduction in microbial diversity concurrent with a high Be level observed in human fecal samples (Additional file 3: Figure S2) can be reproduced by the in vivo mice study (Fig. 5C). Indeed, similar to the human fecal analysis results, Simpson and Shannon diversity indices were significantly decreased in the HFD-Be groups. To assess the variation in gut microbiota between groups and identify features differentially abundant in the HFD-Con and HFD-Be groups, we implemented the random forest machine-learning algorithm (Fig. 5d, e) and found significant decreases in relative abundance of Allobaculum and Akkermansia and increasing trends in Lactobacillus and Oscillospira.

Be affects cecal short chain fatty acids (SCFAs), colonic function, and inflammation.

Next, we evaluated whether Be exposure affects the production of SCFAs-the major microorganismderived metabolites (Fig. 6a). Total SCFA content in the cecum was not significantly different between the groups; however, there were significant changes in the ratio of acetate, propionate, and butyrate, which are the three major SCFAs. Acetate levels increased after Be exposure in the HFD group, while propionate and butyrate levels decreased significantly. Such a result was reasonable because Akkermansia, Coprococcus, and Bifidobacterium, which were reduced after exposure to $\mathrm{Be}$, are the representative propionate and butyrate producers and have a strong butyrogenic effect, respectively. Be exposure also affected colonic mRNA expression in HFD-fed mice (Fig. 6b); Be exposure has markedly increased the expression of the mucin (Muc)2 gene, which is associated with gel-forming mucin production, whereas occludin (Occl) and zonula occludens (Zo)-1 expression-associated with intestinal cell integrity-was not significantly affected. Notably, there was a significant decrease in peptide YY (PYY), the anorexigenic hormone. Moreover, the inflammation-related genes, including inducible nitric oxide synthase (Nos2) and interleukin (IL) $1 \beta$, were highly expressed in the Be groups, whereas IL-10 expression, which is associated with inflammation regulation, was inhibited. We further compared the ratio of Gram-positive (G+) to Gram-negative (G-) bacteria between the groups to determine whether the metabolic abnormalities were 
induced by low-grade inflammation with metabolic endotoxemia. Although the HFD groups showed a significant reduction in the $\mathrm{G}+\mathrm{G}$ - ratio compared with that of the ND groups, there was no clear decrease in the G+/G- ratio in HFD-Be groups based on Be concentration (Additional file 8: Figure S6). We then measured plasma lipopolysaccharide (LPS) levels to assess whether LPS from G- bacteria spilled into systemic circulation and caused metabolic endotoxemia (Fig. 6c) and found that an increase in Be exposure led to a minimal increase in plasma LPS levels that was not significant. Nevertheless, plasma LPS levels in the HFD groups were higher than in the ND groups, and HFD-Be3 and HFD-Be30 showed significant increases compared with that of the ND-Con group, indicating that the combination of HFD and Be exposure may lead to more severe endotoxemia. To examine whether the combined increase in Be and LPS, which may have resulted from mucosal barrier disintegration by HFD, is linked to the induction of inflammatory response, we performed in vitro cell experiments using mouse macrophage-like RAW264.7 cells. We carried out Be treatment with or without LPS to assess the combinatory effects of Be and LPS (Fig. 6d) and found that Be + LPS significantly increased nitrite production compared with that of the LPS only treatment group. Be treatments alone did not increase nitrite production.

\section{Discussion}

In this study, we conducted metallomic analysis to measure fecal element content and performed NMRbased fecal metabolite analysis of human stool samples. Our results underscore the fact that convergence studies using omics data from excreted human feces may be important for innovative data analysis with systemic perspectives that can help in the acquisition of more information regarding the gut environment.

It has been recently revealed that some heavy metals such as $\mathrm{As}, \mathrm{Cd}$, and $\mathrm{Pb}$ may lead to a shift in the gut microbial community and worsen metabolic disorders even at relatively low concentrations [7, 9, 11]. Although not significant, $\mathrm{As}, \mathrm{Cd}$ and $\mathrm{Pb}$ levels have shown a relatively strong positive correlation with MetS in our studies (Fig. 1a). Moreover, As showed a negative relationship with Odoribacter and Parabacteroides abundance, which were abundant in the healthy group compared with the MetS group in our previous study [14] (Fig. 1b). In contrast, Zn, which has been recently linked to improving diabetes via insulin resistance and blood sugar reduction [5], showed the opposite results in the correlation analysis of MetS and gut microbiota (Fig. 1a, b). These findings indicate that the metallomic analysis results are reliable. Furthermore, Be and Ca were the elements highly associated with MetS (Fig. 1a). According to recent studies, Ca supplementation leads to a significant decrease in body weight and adiposity, along with significant increases in Bifidobacterium, Bacteroides, and Akkermansia abundance [17]; this is consistent with our findings on human fecal samples (Fig. 1b). Although the association between $\mathrm{Ca}$ and metabolic disorders has already been reported in some studies [17-19], few have reported on the risk of Be on MetS. We thus evaluated the relationship between Be, the human gut microbiome, and MetS status using an in vivo mice model and found that a low dose of Be exposure can influence gut microbial changes and worsen MetS. This finding is of great significance as it reveals the risk of a low dose of Be on MetS. 
Studies on the health risks of Be have reported that Be exposure is associated with several adverse health outcomes including lung cancer as well as acute and chronic Be disease [20-22]. Because Be toxicity has mostly been reported with regards to the respiratory tract, legal regulations and studies on Be have focused on exposure through inhalation. In this work, we utilized $3 \mathrm{ppb}$ and $30 \mathrm{ppb}$ Be in drinking water to evaluate the effect of low doses of Be exposure via oral intake on the metabolic homeostasis of HFD mice. The daily Be intake level of mice exposed to $30 \mathrm{ppb}$ of Be water was equivalent to $\sim 1.7 \mu \mathrm{g} / \mathrm{kg} / \mathrm{day}$ estimated from the consumption of drinking water, which closely corresponded to the tolerable daily intake (TDI) of $2 \mu \mathrm{g} / \mathrm{kg} /$ day and was much lower than the commonly known no observed adverse effect level (NOAEL) of Be, which is $0.1 \mathrm{mg} / \mathrm{kg} /$ day. Such a low dose of Be was still found to affect body weight gain and worsen MetS symptoms in HFD mice (Fig. 4). In particular, analysis of the gut microbiome and colonic mRNA expression revealed that exposure to both $30 \mathrm{ppb}$ and $3 \mathrm{ppb}$ Be can disturb the microbial community and expression of MetS-related genes in HFD mice (Fig. 5 and Fig. 6). The current drinking water standard for Be established in 1992 by the Environmental Protection Agency (USEPA, 1992) is $4 \mathrm{ppb}$, and a recent WHO report determined that it is not necessary to set a formal guideline value for $\mathrm{Be}$ in drinking water because $\mathrm{Be}$ is rarely found in drinking water at concentrations that warrant health concerns $[23,24]$. However, according to one study on Be exposure, the estimated total daily Be intake of the general US population was $423 \mathrm{ng}$, with the largest contributions being from drinking water (300 ng/day) and food (120 ng/day), with smaller contributions from air and dust ( $2.8 \mathrm{ng} /$ day) [23]. Thus, our results emphasize that more reliable and extensive investigations are required to analyze the occurrence, intake, and toxicity of Be from not only air but also from food and drinking water, and that the current water standard for Be may need to be revised.

Other studies have also evaluated the risk of Be through oral intake $[25,26]$, yet unlike our findings, they reported less than $10 \%$ body weight loss in animal studies. Perhaps this is because the experimental conditions were different from our study; for example, the studies focused on the cancer risk of Be, which was therefore tested at a concentration of more than $5 \mathrm{ppm} \mathrm{Be}$, which is approximately 150 times higher than the maximum concentration (30 ppb) we used. Moreover, they evaluated Be risk under ND conditions, whereas we found that a low dose of Be exposure increased risk under HFD conditions. Notably, other studies found that a low dose of Be exposure increased body weight and feed intake during the first 30 days $[27,28]$. This is consistent with our results where increased feed intake was observed during the beginning stages of the experiment (Fig. 3b). Furthermore, our cell experiments demonstrated the combinatory effects of Be and LPS on the increase in inflammation, suggesting that low doses of Be may be toxic under certain conditions (Fig. 6d). There is a related study that showed how co-treatment with LPS and $100 \mu \mathrm{M}$ Be sulfate (equivalent to $900 \mathrm{ppb} \mathrm{Be}$ ) significantly increased IL-1 $\beta$ and decreased IL-10 compared with that of the LPS only treatment [29]. These findings are consistent with our findings, and even we could obtain a similar result from the low dose of Be treatment that was about 30 times less than the test. Taking all these findings into consideration, the risk of Be exposure may become more severe when mucosal barrier functions are incomplete, such as under HFD conditions or during early developmental stages. 
HFDs increase Gram-negative bacteria abundance and mucosal barrier disintegration, which is followed by LPS spilling into the systemic circulation, causing metabolic endotoxemia with low-grade inflammation [30]. In our study, pro-inflammatory markers such as Nos 2 and IL-1 $\beta$ were highly expressed in HFD-Be groups, whereas the expression of IL-10, which is an anti-inflammatory cytokine associated with inflammation regulation, was significantly reduced (Fig. 6b), indicating that colonic inflammation became worse. In addition, HFD-Be groups showed a significant increase in plasma LPS levels compared with that of the ND-Con group (Fig. 6c). Thus, metabolic endotoxemia and intestinal inflammation markedly increased due to the combination of HFD and Be. What is unusual is that the hyper-expression of the Muc2 gene, which is associated with the release of gel-forming mucin, was observed (Fig. 6b). According to another study, if pro-inflammatory cytokine levels are increased, then Muc2, Muc1, and Muc4 expression increases in goblet cells, after which the continuous Muc1 and Muc4 expression finally promote cancer development [31]. Given that Be is one of the most toxic metals, the hyper-expression of Muc2 may result from the compensatory host defense response against HFD and Be exposure to prevent mucosal barrier impairment. Moreover, HFDs may not only cause disintegration of the mucus barrier but they can also induce a slow transit through the gut. Consequently, this increases the duration of time toxic elements such as Be remain in the gastrointestinal tract, which would have a higher probability of worsening the gut environment. Therefore, HFD-Be groups showed a more pronounced effect on the altered gut microbiota and worsened MetS compared with that of the ND-Be group.

Be exposure led to significant changes in the gut microbiota. It is noteworthy that our human study, in vitro fecal culture, and in vivo mouse experiments showed Be-dependent reduction in the abundance of Akkermansia and Bifidobacterium, which have been heavily studied for their effects on MetS suppression $[15,16]$. In particular, Be exposure significantly decreased the production of propionate and butyrate as well as expression of the anorexigenic PYY (Fig. 6a). Because butyrate and propionate are reported to be predominantly anti-obesogenic, inducing the secretion of anorexigenic hormones such as PYY and glucagon-like peptide (GLP)-1 for appetite regulation [32], and as Akkermansia and Bifidobacterium are known as propionate-producing bacteria [33], these changes appear reasonable. Moreover, acetate ratios were markedly increased in the human Be-high group and the HFD-Be mice groups with a temporary increase in feed consumption during early mouse development (Fig. 4b and Fig. 6a). As the majority of acetate is absorbed into the body where it acts as a substrate for hepatic and adipocyte lipogenesis, acetate is believed to have more obesogenic potential than do propionate and butyrate [32]. In addition, a recent study has shown that high levels of plasma acetate originating from gut microbial dysbiosis can increase glucose-induced insulin secretion to promote the release of ghrelin-an orexigenic hormoneleading to metabolic disease through parasympathetic activation [34]. Thus, Be exposure may lead to worsened MetS concurrent with an increase in appetite resulting from the alteration in gut microbiota.

\section{Conclusions}

Overall, our study indicated that fecal elements are highly associated with gut microbiota and MetS. Exposure to Be may lead to changes in gut microbial composition and worsen MetS even at very low concentrations. Our study is significant for several reasons as analysis of fecal elements and metabolites 
can provide information on environmental factors, including human exposure levels to various elements and chemicals. Moreover, certain gut microbiota were strongly correlated with fecal elements, demonstrating a need for follow-up research on these elements to further elucidate the interaction mechanisms between elements, gut microbiota, and diseases. In particular, we found that a low dose of $\mathrm{Be}$-almost identical to the current global standard levels for drinking water-can be an important cause of MetS by disturbing gut microbiota and inducing inflammation responses in mice fed HFD.

Nevertheless, our study has some inherent limitations when conducting cross-sectional studies. Despite these limitations, certain elements showed significant associations with MetS and gut microbiota. Longitudinal studies will be required for specific cohorts along with large-scale element analysis of additional human cohort for further verification. Moreover, further research is warranted to elucidate the disturbance mechanism of Be on gut microbiota and host metabolism.

\section{Methods}

\section{Human subjects}

This study was approved by the Institutional Review Board of the Seoul National University (IRB No. 1442011-07-11) and was performed according to the Helsinki Declaration. Written informed consent was obtained from each participant. A total of 304 individuals from participants enrolled in the Healthy Twin Study in South Korea [35] were selected for this study. Fecal samples from participants were collected at home and immediately frozen in a home freezer, followed by transfer to clinics and storage at $-80^{\circ} \mathrm{C}$ until further analysis. All participants filled in questionnaires covering lifestyle, medication, disease history, biochemical tests, and anthropometrical measurements. The gut microbiome data were acquired from our previous study (accession number: ERP010289) [14]. The demographic characteristics of the study subjects are listed in Additional file 9: Table S3.

Measurement of MetS components and definition of MetS

Measurements of waist circumference, blood pressure, triglyceride, HDL cholesterol, and FBS have been previously published [14]. MetS was defined following the revised National Cholesterol Education Program Adult Treatment Panel III criteria with the Korean-specific waist circumference cut-off values for abdominal obesity. The subjects were considered to have MetS if they had three or more of the following five criteria, (1) waist circumference $\geq 90 \mathrm{~cm}$ for men or $\geq 85 \mathrm{~cm}$ for women, (2) blood pressure $\geq$ $130 / 85 \mathrm{~mm} \mathrm{Hg}$, (3) triglycerides $\geq 150 \mathrm{mg} / \mathrm{dL}$ ( $1.7 \mathrm{mmol} / \mathrm{L})$, (4) HDL cholesterol < $40 \mathrm{mg} / \mathrm{dL}(1.03$ $\mathrm{mmol} / \mathrm{L})$ for men or $<50 \mathrm{mg} / \mathrm{dL}(1.3 \mathrm{mmol} / \mathrm{L})$ for women, and (5) FBS $\geq 100 \mathrm{mg} / \mathrm{dL}(5.6 \mathrm{mmol} / \mathrm{L})$.

In vitro batch culture of human fecal microbiota

In vitro colonic fermentation was performed according to Long's method with minor modification [36]. Briefly, $8 \mathrm{~g} / \mathrm{L} \mathrm{NaCl}, 1.15 \mathrm{~g} / \mathrm{L} \mathrm{Na}_{2} \mathrm{HPO}_{4}, 0.5 \mathrm{~g} / \mathrm{L} \mathrm{L-cysteine,} 0.2 \mathrm{~g} / \mathrm{L} \mathrm{KCl}$, and $0.2 \mathrm{~g} / \mathrm{L} \mathrm{KH}_{2} \mathrm{PO}_{4}$ were dissolved in distilled water and autoclaved for making PBS medium. Fecal samples were obtained from three healthy donors (age 20-30; mean BMI 22.3) who had taken no antibiotics or prebiotics for three months 
prior to the study. Written informed consent was obtained from donors, and the study was approved by the institutional review board of the Korea Institute of Science and Technology (IRB No. 2015-003). Fecal slurry $(10 \% \mathrm{w} / \mathrm{v})$ was prepared by diluting and suspending the fecal samples with PBS medium in an anaerobic chamber (Coy Laboratory Products Inc., Ann Arbor, MI). The cultivation was started with 5\% fecal inoculum by adding $0.9 \mathrm{~mL}$ of $10 \%$ fecal slurry into $0.9 \mathrm{~mL}$ PBS medium (total volume, $20 \mathrm{~mL}$ ) using a 96-deep well plate. Culturing was performed with various concentrations of $\mathrm{Be}(3,30,300$, and $3000 \mathrm{ppb}$ ) in an anaerobic jar (MGC, Japan) with AnaeroPack (MGC) at $37^{\circ} \mathrm{C}$ without stirring. Samples were collected after $24 \mathrm{~h}$ and stored in the refrigerator $\left(-20^{\circ} \mathrm{C}\right)$ for further analysis.

\section{Animals and exposure}

All animal experiments were conducted with the approval of the Institutional Animal Care and Use Committee of the Korean Institute of Science and Technology (No. 2018-036) and strictly followed National Institutes of Health guidelines for the use of live animals. Male C57BL/ 6 mice (5-week-old, 20$22 \mathrm{~g}$; Central Lab. Animal Inc., Seoul, South Korea) were housed in individually ventilated cages at $23 \pm$ $0.5^{\circ} \mathrm{C}$ and $10 \%$ humidity under a 12-h light-dark cycle with ad libitum access to feed and water. All animals were acclimated for 7 days and separated into 2-3 animals/cage, ensuring equal weight average. Be was administered to mice as beryllium sulfate tetrahydrate (Sigma-Aldrich, St. Louis, MO) in drinking water for 50 days. Freshly prepared Be-containing water ( 3 or $30 \mathrm{ppb}$ ) and feed were provided to mice twice a week, while control mice received feed with water alone. Mice were randomly divided into three HFD groups and two ND groups as follows, (1) HFD (45\% of total calories from fat; TD.06415; Harlan Laboratories, Indianapolis, IN) with water $(n=8)$, (2) HFD with $3 \mathrm{ppb}$ of Be water $(n=8)$, (3) HFD with $30 \mathrm{ppb}$ of Be water $(n=8)$, (4) ND with water $(n=6)$, and (5) ND with $30 \mathrm{ppb}$ of Be water $(n=6)$. Feed and water intake were recorded once a week for each cage and the data were used for the calculation of average intake per mouse per week. The theoretical weekly Be exposure was calculated by multiplying the added Be concentration by the average weekly water intake. Body weight of each mouse was measured once a week and stool samples were collected once a week and immediately stored at -80 ${ }^{\circ} \mathrm{C}$ for further analysis. Animals were euthanized by $\mathrm{CO}_{2}$ inhalation at the beginning of the light cycle and after $16 \mathrm{~h}$ of food deprivation. Blood samples were collected by cardiac puncture in microtubes containing EDTA and centrifuged at $1,000 \times \mathrm{g}$ and $4^{\circ} \mathrm{C}$ for $15 \mathrm{~min}$ to obtain plasma, and stored at $-80{ }^{\circ} \mathrm{C}$ for subsequent biochemical measurements. The eWAT, cecum, and colon of each mouse were precisely dissected, weighed, and stored for further analysis. All tissues were rinsed with saline and snap-frozen at $-80^{\circ} \mathrm{C}$

16S rRNA gene sequence analysis

DNA was extracted from stools, in vitro batch culture samples, and mice cecum using a QIAamp DNA Stool Mini Kit (Qiagen, Hilden, Germany) with an additional bead-beating procedure to improve DNA recovery for Gram-positive bacteria. The $16 \mathrm{~S}$ rRNA genes were amplified using an improved dual-indexing amplification of the V3-V4 region (319F/806R) of the 16S rRNA gene with a heterogeneity spacer [37]. PCR products were purified using AMPure XT beads (Beckman Coulter, Danvers, MA) and quantified 
using a Qubit dsDNA high-sensitivity reagent (Invitrogen, Carlsbad, CA). Sequencing was conducted on the MiSeq platform using a paired-end $2 \times 300$-bp reagent kit (Illumina, San Diego, CA). The raw reads were demultiplexed, assembled, and quality-filtered in QIIME 2 (v2018.6) using the default settings. DADA2 was used to filter chimeric reads and artifacts commonly present in Illumina amplicon data [38]. To classify filtered reads to taxonomic groups, a Naive Bayes classifier was trained using the 16S rRNA region (V3-V4), the primer set and read length used (319F/806R, 469 bp), and the Greengenes $99 \%$ reference set (v13.8) [39]. This trained feature classifier was then used to assign taxonomy to each read using the default settings in QIIME. Microbial composition at a certain level as well as a- and $\beta$-diversity were analyzed using MicrobiomeAnalyst [40]. Non-metric multidimensional scaling (NMDS) plots were generated from a Bray-Curtis distance matrix, and a principal coordinate analysis (PCoA) plot was generated using unweighted Unifrac distances to visually represent microbiota compositional differences among groups. Random forest, a supervised learning method for the classification of human microbiome data [41], was used to select subsets of taxa (genus level) that are highly discriminative of the type of community from Be-exposed mice. We measured feature importance as the mean decrease in model accuracy when that feature's values were permuted randomly using 500 trees and seven repetitions.

Microbial functional analysis

Phylogenetic investigation of communities by reconstruction of unobserved states (PICRUSt) was used to infer putative functional metagenomes from 16S rRNA gene sequence profiles [42]. As the tool adapts OTUs with Greengene IDs, OTUs were picked with closed reference against the May 2013 Greengenes database. The relative abundance of each functional pathway was obtained for each sample, and nonmicrobial functional pathways belonging to the "Organismal Systems" and "Human Diseases" categories were excluded from downstream analysis. To determine metabolic features that were differentially abundant between each element status (low and high level), linear discriminant analysis effect size (LEfSe) was applied under the condition $a=0.05$, with an LDA score of at least two [43].

Fecal elements analysis with inductively coupled plasma mass spectrometry (ICP-MS)

ICP-MS calibration standard solutions were prepared from $10 \mathrm{mg} / \mathrm{L}$ multi-element standard solution (PerkinElmer, Waltham, MA). The standard calibration solutions were prepared by dilution of the standard solutions with a suitable percentage of analytical grade concentrated $\mathrm{HNO}_{3}$ (Dongwoo Fine Chem.Co., Ltd., South Korea). De-ionized water (18.2 M $\Omega-\mathrm{cm})$ was prepared by a Milli-Q water purification system (Millipore, Bedford, MA). All chemicals and reagents used in this experiment were obtained from SigmaAldrich and of analytical reagent grade unless otherwise stated. Before use, all plastic and glassware were soaked in $10 \%$ nitric acid for at least $24 \mathrm{~h}$ and then rinsed with deionized water several times. To measure the total metal concentration in the samples, microwave-assisted acid digestion was performed. Fecal samples $(\sim 0.5 \mathrm{~g})$ were weighed directly into quartz microwave digestion tubes and then $4.0 \mathrm{~mL}$ concentrated $\mathrm{HNO}_{3}$ was added. The $1.5-\mathrm{kW}$ microwave was used to reach $230{ }^{\circ} \mathrm{C}$ within 20 min and the temperature was maintained for 15 min with a microwave digestion system (UltraWAVE; Milestone srl, Sorisole, Italy), followed by cooling without a microwave. The tube contents were then transferred to 
polypropylene tubes and diluted to $50.0 \mathrm{~g}$ with deionized water. Sample blanks were prepared by following the above procedure. ICP-MS measurements were performed using a quadrupole ELAN DRC-e spectrometer (Perkin-Elmer SCIEX, Norwalk, CT) equipped with a concentric nebulizer (Meinhard Associates, Golden, CO), a cyclonic spray chamber (Glass Expansion, Inc., West Melbourne, Australia), a quartz torch with a quartz injector tube ( $2 \mathrm{~mm}$ i.d.), and an autosampler (AS-93 Plus, Perkin-Elmer) for the simultaneous determination of metals. The following operational conditions were used, radiofrequency power of $1.4 \mathrm{~kW}$ and plasma, auxiliary, and nebulizer gas flow rates of $16,1.2$, and $0.9 \mathrm{~L} / \mathrm{min}$, respectively. Quality control was conducted throughout sample analysis. Quantitative analysis of the samples was performed by external calibration. To monitor the consistency of the instruments, the calibration standards were analyzed as samples regularly. Continuous calibration verification (CCV) standard solutions were measured after every ten samples. Data were accepted only when CCV samples were $90-110 \%$ of the expected value. Deionized water blanks were also analyzed at regular intervals to check for cross-contamination or losses.

NMR-based metabolomic analysis

For NMR-based metabolomic analysis, samples were prepared according to Lamichhane's method with minor modifications [44]. Briefly, human fecal samples ( 200 mg) were mixed with $1000 \mu \mathrm{L} D D W$, vortexed for $30 \mathrm{~s}$ and homogenized with a tissue homogenizer for $5 \mathrm{~min}$. After centrifugation $(14,000 \times g$, $\left.4^{\circ} \mathrm{C}\right)$ for $10 \mathrm{~min}, 300 \mu \mathrm{L}$ of supernatant was mixed with $60 \mu \mathrm{L}$ deuterium oxide $\left(\mathrm{D}_{2} \mathrm{O}\right)$ containing 0.025 $\mathrm{mg} / \mathrm{mL}$ 3-(trimethylsilyl) propionic acid-d4 sodium salt, $60 \mu \mathrm{L}$ of $1 \mathrm{mM}$ imidazole, $60 \mu \mathrm{L}$ of $2 \mathrm{mM} \mathrm{NaN3}$, and $120 \mu \mathrm{L}$ of $0.5 \mathrm{M} \mathrm{KH}_{2} \mathrm{PO}_{4}$. The mixtures were vortexed for $1 \mathrm{~min}$ and centrifuged at $14,000 \times g$ for 10 min. The clear supernatant was then transferred to a $5 \mathrm{~mm}$ NMR tube (Wilmad-LabGlass, Vineland, NJ) for NMR analysis. All ${ }^{1} \mathrm{H}$-NMR spectra were acquired using a Varian $500 \mathrm{MHz}$ NMR system (Varian, Palo Alto, CA) equipped with a cold flow-probe. ${ }^{1} \mathrm{H}$-NMR spectra were collected at $25^{\circ} \mathrm{C}$ using the water presaturation pulse sequence. Spectra were collected with 64 transients using a $4 \mathrm{~s}$ acquisition time and a $2 \mathrm{~s}$ recycle delay. Tentative assignments of ${ }^{1} \mathrm{H}$ NMR signals were carried out using the Bayesian automated metabolite analyzer for NMR (BATMAN) and confirmed by Chenomx NMR Suite 8.3 (Chenomx Inc, Alberta, Canada) according to the Human Metabolome Database. A total of 67 metabolites (acetate, acetoin, alanine, arginine, aspartate, betaine, butyrate, cadaverine, carnitine, carnosine, cholate, choline, creatine, cysteine, folate, formate, fructose, fucose, fumarate, galactose, glucose, glutarate, glyceraldehyde, glycerol, glycine, heptanoate, histamine, histidine, hypoxanthine, indole, indoxyl sulfate, isobutyrate, isoleucine, isovalerate, lactate, leucine, lysine, malate, malonate, mannose, methionine, methyl succinate, $\mathrm{N}$-acetylglutamate, $\mathrm{N}$-acetylneuraminate, proline, propionate, purine, putrescine, pyrimidine, pyruvate, ribose, sarcosine, succinate, threonine, thymine, trimethylamine, trimethylamine oxide, tryptophan, tyrosine, uracil, urocanate, valerate, valine, xanthine, xylose, $y$-aminobutyrate, and $\rho$ cresol) were assigned for analysis. The metabolomic data were imported into MetaboAnalyst 4.0 and normalized for multivariate pattern recognition analysis [45]. Partial least squares discriminant analysis (PLS-DA) was performed to obtain an overview of the complete metabolomic data set after mean 
centering scaling. Variable importance of projection (VIP) scores were assessed to rank the differential metabolites among each element status.

\section{Biochemical measurements}

Plasma triglyceride (DoGEN Bio Co., Ltd, Seoul, South Korea), glucose (Abcam, Cambridge, UK), insulin (Abcam), leptin (Abcam), and adiponectin (Abcam) concentrations were measured using commercial ELISA kits according to the manufacturer's instructions. LPS levels were detected using an endpoint chromogenic endotoxin quantitative test (Signalway Antibody, College Park, MD).

\section{SCFA measurement}

Cecal SCFAs content was determined by gas chromatography. Cecal contents ( 80 mg) were homogenized in $500 \mu \mathrm{L}$ deionized water, after which the samples were acidified with $50 \mu \mathrm{L} 50 \%$ sulfuric acid, followed by vortexing at room temperature for $5 \mathrm{~min}$. After centrifugation at $14,000 \times g$ for $10 \mathrm{~min}$, $400 \mu \mathrm{L}$ of the supernatant was transferred to a new tube, and $40 \mu \mathrm{L}$ internal standard (1\% 2-methyl pentanoic acid) and $400 \mu \mathrm{L}$ anhydrous ethyl ether were added. The tube was vortexed for $1 \mathrm{~min}$ and then centrifuged at $14,000 \times g$ for $10 \mathrm{~min}$. The upper ether layer was used for further analysis. Volatile Free Acid Mix (Sigma-Aldrich) was used as the SCFA standard for the quantification of acetate, butyrate, isobutyrate, propionate, valerate, and isovalerate. GC-FID (GC 450; Bruker, Billerica, MA) was used to analyze SCFA content with fused silica capillary columns (Nukol, $30 \mathrm{~m} \times 0.25 \mathrm{~mm}, 0.25-\mu \mathrm{m}$ film thickness). The oven temperature was $170{ }^{\circ} \mathrm{C}$, and the FID and injection ports were set to $225^{\circ} \mathrm{C}$. Nitrogen was used as the carrier gas and the sample injection volume was $2 \mu \mathrm{L}$.

RNA extraction and real-time PCR analysis

Total RNA was extracted from colon tissues ( $50 \mathrm{mg})$ using TRIzol reagent (Thermo Fisher Scientific, Waltham, MA) according to the manufacturer's instructions, followed by concentration measurement. cDNA was synthesized from $1 \mu \mathrm{g}$ of total RNA using Superscript IV Reverse Transcriptase (Thermo Fisher Scientific). Real-time PCR was performed using the LightCycler 480 detection system (Roche Diagnostics, Rotkreuz, Switzerland) and LightCycler 480 SYBR Green I Master; for primer sequences, see Appendix E. Samples were run in duplicate in a single 384-well reaction plate. Data were normalized to the housekeeping RPL19 gene and analyzed according to the $\Delta \Delta \mathrm{CT}$ method.

\section{Cell culture}

The mouse macrophage cell line RAW264.7 was maintained in Dulbecco's modified Eagle's medium (Thermo Fisher Scientific) supplemented with 10\% fetal bovine serum (Thermo Fisher Scientific) and $1 \%$ ( $\mathrm{v} / \mathrm{v})$ penicillin $(100 \mathrm{U} / \mathrm{mL})$-streptomycin $(100 \mu \mathrm{g} / \mathrm{mL})$ (Thermo Fisher Scientific). Cells were grown in 75 $\mathrm{cm}^{2}$ tissue culture flasks and incubated at $37{ }^{\circ} \mathrm{C}$ and $5 \% \mathrm{CO}_{2}$. Cells were detached from the flask with a scraper and split 1:10 every five days. For nitrite measurement experiments, RAW264.7 cells $\left(5 \times 10^{4}\right.$ cells/well) were seeded in 24-well plates with $1 \mathrm{~mL}$ of medium per well. Cells were treated with LPS (100 
$\mathrm{ng} / \mathrm{mL})$ as a positive control and a series of concentrations of $\mathrm{Be}(3,30$, and $100 \mathrm{ppb})$ after $24 \mathrm{~h}$ of inoculation. Fecal microbial supernatants and cell pellets from $1 \mathrm{~mL}$ of in vitro anaerobic culture with various concentrations of $\mathrm{Be}(3,30$, and $100 \mathrm{ppb})$ were also used to treat RAW264.7 cells.

\section{Determination of NO production}

The nitrite concentration in the culture medium was measured as an indicator of NO production using the Griess reaction. After incubation with test samples for $48 \mathrm{~h}$, the supernatant from each well $(50 \mu \mathrm{L})$ was transferred to a fresh 96-well plate, after which $25 \mu \mathrm{L}$ of $1 \%$ sulfanilamide and $25 \mu \mathrm{L}$ of $0.1 \%$ naphthylethylenediamine in $5 \% \mathrm{HCl}$ was added. After $10 \mathrm{~min}$ of incubation at room temperature, the absorbance of each well was measured at $540 \mathrm{~nm}$ using a Synergy HT microplate reader (Biotek, Winooski, VT). Relative nitrite production was calculated relative to the LPS only treatment group.

Statistical and subsequent bioinformatics analysis

Statistical analysis of all grouped data was performed using R software or GraphPad Prism 7 (GraphPad Software Inc., La Jolla, CA). Significance was determined using a two-tailed Student's $t$ test, MannWhitney test, or one-way ANOVA corrected for multiple comparisons with a Sidak test compared with the control group. Microbial data processing and multivariate statistical analysis were performed using MicrobiomeAnalyst. Permutational multivariate analysis of variance (PERMANOVA) was performed to test the association between microbiome composition and Be exposure based on NMDS. Association of fecal elements with MetS-related clinical biomarkers and gut microbiota was assessed by Spearman's rank correlation analysis. A correlation heatmap was generated using the R package "Pheatmap." $P$ values were adjusted for multiple testing with the Benjamini-Hochberg method. Multivariate analysis using a multivariate association with linear models (MaAsLin) was performed to identify significant associations of microbial abundances with metabolic status or fecal element status [46]. Age, sex, and smoking were treated as fixed effects, while MZ, DZ twin, and family relationships were treated as random variables. Low abundance taxa (the average relative abundance across all the samples $<0.1 \%$ ) were excluded from MaAsLin analysis.

\section{Declarations}

\section{Ethics approval and consent to participate}

This study was approved by the Institutional Review Board of the Seoul National University and was performed according to the Helsinki Declaration. Written informed consent was obtained from each participant. All animal experiments were conducted with the approval of the Institutional Animal Care and Use Committee of the Korean Institute of Science and Technology and strictly followed National Institutes of Health guidelines for the use of live animals.

\section{Consent for publication}

Not applicable 
Availability of data and material

All raw data of $16 \mathrm{~S}$ rRNA gene sequencing are available at Sequence Read Archive (Accession number SUB6849454).

\section{Competing interests}

We declare that we have no competing interests.

\section{Funding}

This research was supported by the National Research Foundation of Korea (NRF2018R1A2A1A05078258), the Bio \& Medical Technology Development Program of the National Research Foundation funded by the Ministry of Science \& ICT (NRF-2016M3A9D3915857), and an intramural grant (2Z05620) from the Korea Institute of Science and Technology.

\section{Authors' contributions}

This project was designed by K.H.C. and J.S.Y. Element analysis was performed by J.S.Y. Metabolomic analysis was performed by K.H.C. and J.B.J. Metagenomic data were generated by M.Y.L., H.S.Y., K.H.C., and K.Y.Y. and analyzed by M.Y.L., B.S. and K.H.C. Mice experiments were performed by K.A.K., D.G.S., K.Y.Y., E.E., and K.H.C. with the assistance of H.J.Y. B.S. and H.J.Y. collected human stool. C.H.P and G.K. managed and supported this project.

\section{Acknowledgments}

We thank Prof. K. Choi, S. Kim, and J. B. Kim from Seoul National University for their assistance and contributions during this research.

\section{References}

1. Bhurosy T, Jeewon R. Overweight and obesity epidemic in developing countries: a problem with diet, physical activity, or socioeconomic status? ScientificWorldJournal. 2014;2014:964236.

2. Holtcamp W. Obesogens: an environmental link to obesity. Environ Health Perspect. 2012;120:a62-8.

3. Griffin NW, Ahern PP, Cheng J, Heath AC, Ilkayeva O, Newgard CB, Fontana L, Gordon JI. Prior dietary practices and connections to a human gut microbial metacommunity alter responses to diet interventions. Cell Host Microbe. 2017;21:84-96.

4. Nielsen FH. Evolutionary events culminating in specific minerals becoming essential for life. Eur $\mathrm{J}$ Nutr. 2000;39:62-6.

5. Miao X, Sun W, Fu Y, Miao L, Cai L. Zinc homeostasis in the metabolic syndrome and diabetes. Front Med. 2013;7:31-52. 
6. Li P, Fan C, Lu Y, Qi K. Effects of calcium supplementation on body weight: a meta-analysis. Am J Clin Nutr. 2016;104:1263-73.

7. Navas-Acien A, Umans JG, Howard BV, Goessler W, Francesconi KA, Crainiceanu CM, Silbergeld EK, Guallar E. Urine arsenic concentrations and species excretion patterns in American Indian communities over a 10-year period: the Strong Heart Study. Environ Health Perspect. 2009;117:142833.

8. Planchart A, Green A, Hoyo C, Mattingly CJ. Heavy Metal Exposure and Metabolic Syndrome: Evidence from Human and Model System Studies. Curr Environ Health Rep. 2018;5:110-24.

9. Lu K, Abo RP, Schlieper KA, Graffam ME, Levine S, Wishnok JS, Swenberg JA, Tannenbaum SR, Fox JG. Arsenic exposure perturbs the gut microbiome and its metabolic profile in mice: an integrated metagenomics and metabolomics analysis. Environ Health Perspect. 2014;122:284-91.

10. Thingholm LB, Ruhlemann MC, Koch M, Fuqua B, Laucke G, Boehm R, Bang C, Franzosa EA, Hubenthal M, Rahnavard A et al. Obese Individuals with and without Type 2 Diabetes Show Different Gut Microbial Functional Capacity and Composition. Cell Host Microbe. 2019.

11. Zhang S, Jin Y, Zeng Z, Liu Z, Fu Z. Subchronic Exposure of Mice to Cadmium Perturbs Their Hepatic Energy Metabolism and Gut Microbiome. Chem Res Toxicol. 2015;28:2000-9.

12. Ley RE, Backhed F, Turnbaugh P, Lozupone CA, Knight RD, Gordon JI. Obesity alters gut microbial ecology. Proc Natl Acad Sci U S A. 2005;102:11070-5.

13. Turnbaugh PJ, Ley RE, Mahowald MA, Magrini V, Mardis ER, Gordon JI. An obesity-associated gut microbiome with increased capacity for energy harvest. Nature. 2006;444:1027-31.

14. Lim MY, You HJ, Yoon HS, Kwon B, Lee JY, Lee S, Song YM, Lee K, Sung J, Ko G. The effect of heritability and host genetics on the gut microbiota and metabolic syndrome. Gut. 2017;66:1031-38.

15. Hidalgo-Cantabrana C, Sanchez B, Milani C, Ventura M, Margolles A, Ruas-Madiedo P. Genomic overview and biological functions of exopolysaccharide biosynthesis in Bifidobacterium spp. Appl Environ Microbiol. 2014;80:9-18.

16. Dao MC, Everard A, Aron-Wisnewsky J, Sokolovska N, Prifti E, Verger EO, Kayser BD, Levenez F, Chilloux $\mathrm{J}$, Hoyles $\mathrm{L}$ et al. Akkermansia muciniphila and improved metabolic health during a dietary intervention in obesity: relationship with gut microbiome richness and ecology. Gut. 2016;65:426-36.

17. Chaplin A, Parra P, Laraichi S, Serra F, Palou A. Calcium supplementation modulates gut microbiota in a prebiotic manner in dietary obese mice. Mol Nutr Food Res. 2016;60:468-80.

18. Foroozanfard F, Jamilian M, Bahmani F, Talaee R, Talaee N, Hashemi T, Nasri K, Asemi Z, Esmaillzadeh A. Calcium plus vitamin D supplementation influences biomarkers of inflammation and oxidative stress in overweight and vitamin D-deficient women with polycystic ovary syndrome: a randomized double-blind placebo-controlled clinical trial. Clin Endocrinol (Oxf). 2015;83:888-94.

19. Nadeem Aslam M, Bassis CM, Zhang L, Zaidi S, Varani J, Bergin IL. Calcium Reduces Liver Injury in Mice on a High-Fat Diet: Alterations in Microbial and Bile Acid Profiles. PLoS One. 2016;11:e0166178.

20. Administration OSaH. Occupational exposure to beryllium. Final rule. Fed Regist. 2017;82:2470-757. 
21. Huang L, Wang S, Luo J, Zhang Z. Study on high permeabilized opening of lung mitochondrial permeability transition pore and apoptosis induced by beryllium sulfate in mice. J Toxicol. 2015.

22. Mack DG, Falta MT, McKee AS, Martin AK, Simonian PL, Crawford F, Gordon T, Mercer RR, Hoover MD, Marrack $P$ et al. Regulatory T cells modulate granulomatous inflammation in an HLA-DP2 transgenic murine model of beryllium-induced disease. Proc Natl Acad Sci U S A. 2014;111:8553-8.

23. Vaessen HA, Szteke B. Beryllium in food and drinking water-a summary of available knowledge. Food Addit Contam. 2000;17:149-59.

24. Organization WH: Guidelines for drinking-water quality: Fourth edition incorporating the first addendum. Geneva: World Health Organization; 2017.

25. Matsumoto A, Hisada Y, Yoshimura Y. Calcium and phosphate concentrations, and alkaline and acid phosphatase activities in serum of the rat fed with low calcium and beryllium diets. $J$ Oral Ther Pharmacol. 1991;10:253-59.

26. Guyatt BL, Kay HD, Branion HD. Beryllium “Rickets”. The Journal of Nutrition. 1933;6:313-24.

27. Schroeder HA, Mitchener M. Life-term studies in rats: effects of aluminum, barium, beryllium, and tungsten. J Nutr. 1975;105:421-7.

28. Freundt KJ, Ibrahim HA. Growth of rats during a subchronic intake of the heavy metals $\mathrm{Pb}, \mathrm{Cd}, \mathrm{Zn}$, Mn, Cu, Hg, and Be. Pol J Occup Med. 1990;3:227-32.

29. Silva S, Ganguly K, Fresquez TM, Gupta G, McCleskey TM, Chaudhary A. Beryllium alters lipopolysaccharide-mediated intracellular phosphorylation and cytokine release in human peripheral blood mononuclear cells. J Occup Environ Hyg. 2009;6:775-82.

30. Cani PD, Bibiloni R, Knauf C, Waget A, Neyrinck AM, Delzenne NM, Burcelin R. Changes in gut microbiota control metabolic endotoxemia-induced inflammation in high-fat diet-induced obesity and diabetes in mice. Diabetes. 2008;57:1470-81.

31. Kufe DW. Mucins in cancer: function, prognosis and therapy. Nat Rev Cancer. 2009;9:874-85.

32. Shoaie S, Karlsson F, Mardinoglu A, Nookaew I, Bordel S, Nielsen J. Understanding the interactions between bacteria in the human gut through metabolic modeling. Sci Rep. 2013;3:2532.

33. Reichardt N, Duncan SH, Young P, Belenguer A, McWilliam Leitch C, Scott KP, Flint HJ, Louis P. Phylogenetic distribution of three pathways for propionate production within the human gut microbiota. ISME J. 2014;8:1323-35.

34. Perry RJ, Peng L, Barry NA, Cline GW, Zhang D, Cardone RL, Petersen KF, Kibbey RG, Goodman AL, Shulman GI. Acetate mediates a microbiome-brain-beta-cell axis to promote metabolic syndrome. Nature. 2016;534:213-7.

35. Sung J, Cho SI, Lee K, Ha M, Choi EY, Choi JS, Kim H, Kim J, Hong KS, Kim Y et al. Healthy Twin: a twin-family study of Korea-protocols and current status. Twin Res Hum Genet. 2006;9:844-8.

36. Long W, Xue Z, Zhang Q, Feng Z, Bridgewater L, Wang L, Zhao L, Pang X. Differential responses of gut microbiota to the same prebiotic formula in oligotrophic and eutrophic batch fermentation systems. Sci Rep. 2015;5:13469. 
37. Fadrosh DW, Ma B, Gajer P, Sengamalay N, Ott S, Brotman RM, Ravel J. An improved dual-indexing approach for multiplexed 16S rRNA gene sequencing on the Illumina MiSeq platform. Microbiome. 2014;2:6.

38. Callahan BJ, McMurdie PJ, Rosen MJ, Han AW, Johnson AJ, Holmes SP. DADA2: High-resolution sample inference from Illumina amplicon data. Nat Methods. 2016;13:581-3.

39. DeSantis TZ, Hugenholtz P, Larsen N, Rojas M, Brodie EL, Keller K, Huber T, Dalevi D, Hu P, Andersen GL. Greengenes, a chimera-checked 16S rRNA gene database and workbench compatible with ARB. Appl Environ Microbiol. 2006;72:5069-72.

40. Dhariwal A, Chong J, Habib S, King IL, Agellon LB, Xia J. MicrobiomeAnalyst: a web-based tool for comprehensive statistical, visual and meta-analysis of microbiome data. Nucleic Acids Res. 2017;45:W180-W88.

41. Knights D, Costello EK, Knight R. Supervised classification of human microbiota. FEMS Microbiol Rev. 2011;35:343-59.

42. Langille MG, Zaneveld J, Caporaso JG, McDonald D, Knights D, Reyes JA, Clemente JC, Burkepile DE, Vega Thurber RL, Knight $\mathrm{R}$ et al. Predictive functional profiling of microbial communities using $16 \mathrm{~S}$ rRNA marker gene sequences. Nat Biotechnol. 2013;31:814-21.

43. Segata N, Izard J, Waldron L, Gevers D, Miropolsky L, Garrett WS, Huttenhower C. Metagenomic biomarker discovery and explanation. Genome Biol. 2011;12:R60.

44. Lamichhane S, Yde CC, Schmedes MS, Jensen HM, Meier S, Bertram HC. Strategy for NuclearMagnetic-Resonance-based metabolomics of human feces. Anal Chem. 2015;87:5930-7.

45. Chong J, Soufan O, Li C, Caraus I, Li S, Bourque G, Wishart DS, Xia J. MetaboAnalyst 4.0: towards more transparent and integrative metabolomics analysis. Nucleic Acids Res. 2018;46:W486-W94.

46. Morgan XC, Tickle TL, Sokol H, Gevers D, Devaney KL, Ward DV, Reyes JA, Shah SA, LeLeiko N, Snapper SB et al. Dysfunction of the intestinal microbiome in inflammatory bowel disease and treatment. Genome Biol. 2012;13:R79.

\section{Additional Files}

Additional file 1: Figure S1. Schematic diagram illustrating the experimental design of the study.

Additional file 2: Table S1. Fecal levels of 28 elements in healthy and MetS groups. Values are mean \pm $95 \%$ confidence interval.

Additional file 3: Figure S2. a-diversity indices of gut microbiota according to fecal element status. Shannon and Simpson diversity indices were calculated to investigate the a-diversity of the microbial community. The low, middle, and high level groups of Be were determined using the tertile values of the relative abundance of elements. Different letters indicate significant differences (one-way ANOVA and Duncan's tests; $P<0.05 ; n=304)$. 
Additional file 4: Table S2. Significant associations between Be and Ca with microbial taxa by multivariate analysis using MaAsLin. Associations were considered significant with a Benjamini and Hochberg false discovery rate (FDR)-corrected $P$ value of $<0.05$.

Additional file 5: Figure S3. Weekly water intake and Be exposure from drinking water in ND and HFD mice. (A) Water intake at 1, 3, 5, and 7 weeks and (B) theoretical weekly Be exposure. Data represent the means \pm SEM.

Additional file 6: Figure S4. Effect of Be exposure on ND mice host metabolism. (A) Epididymal white adipose tissue mass (eWAT), (B) plasma triglycerides (TGs), (C) plasma glucose, (D) plasma insulin, (E) plasma leptin, and (F) plasma adiponectin levels in control and Be-treated mice ( $n=6 /$ group). Data represent the mean \pm SD. Significance was calculated using unpaired two-tailed Student's $t$ test. ${ }^{\star \star} P<$ 0.01 .

Additional file 7: Figure S5. NMDS plot of the cecal microbiota of Be-exposed ND-fed mice. Bray-Curtis distance matrix was calculated from the genus-level relative abundance data. Significance was determined using PERMANOVA.

Additional file 8: Figure S6. The in vivo mouse ratios of Gram-positive/Gram-negative bacteria. Box plots show the median (horizontal line), mean (cross) and IQR; whiskers represent the minimum and maximum values. Significance was determined using two-tailed Student's $t$ test or one-way ANOVA corrected for multiple comparisons with a Sidak test vs. ND-Con or HFD-Con groups. ${ }^{*} P<0.05$; NS, no significant difference.

Additional file 9: Table S3. Characteristics of the human study population. BP, blood pressure; DZ, dizygotic; F, female; FBS, fasting blood sugar; HDL, high-density lipoprotein cholesterol; M, male; MetS, metabolic syndrome; MZ, monozygotic; Non-twin, parents or siblings of twin pairs; waist, waist circumference. Values are mean $\pm \mathrm{SD}$ or $n(\%) .{ }^{a}$ Abnormal values for at least three of the following, waist, $B P$, triglyceride, $H D L$, and FBS.

\section{Figures}




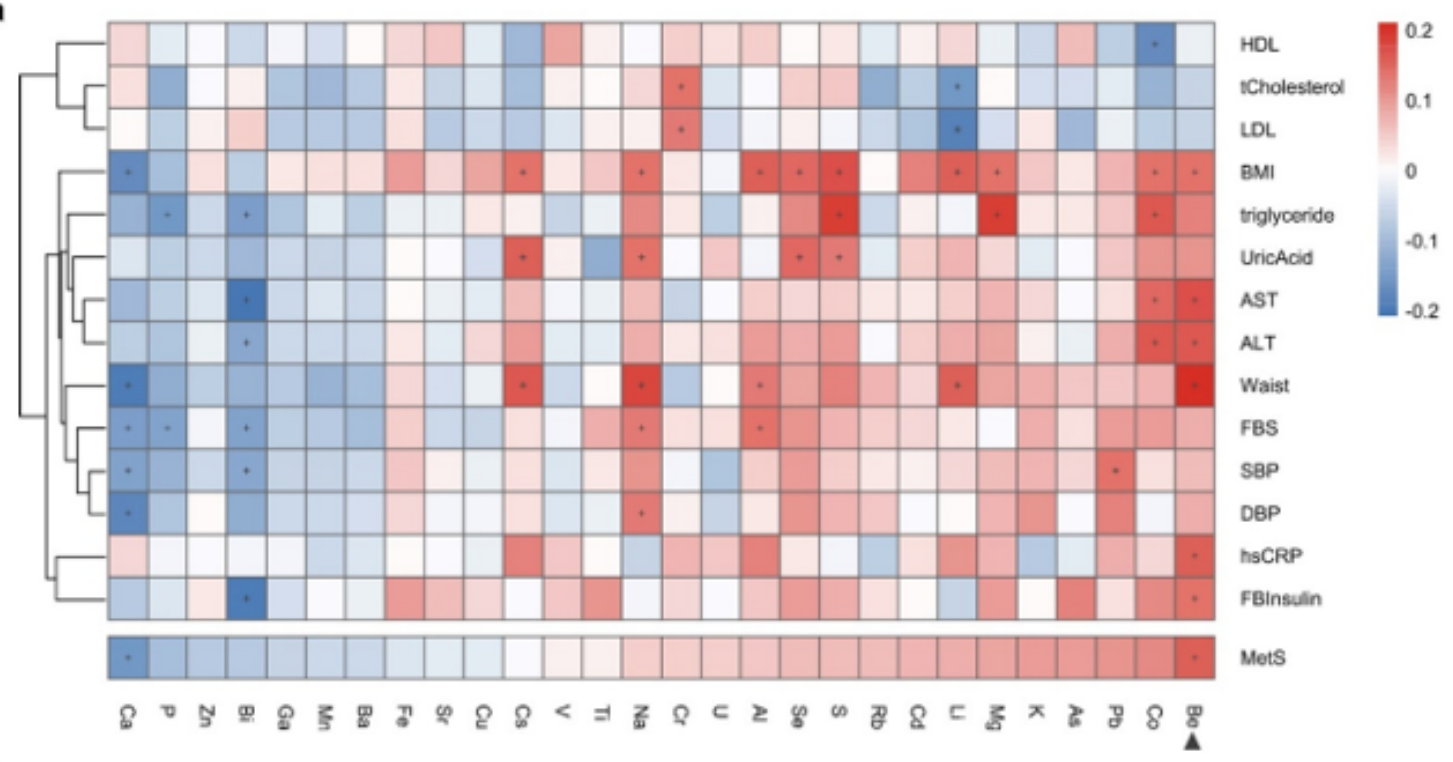

b

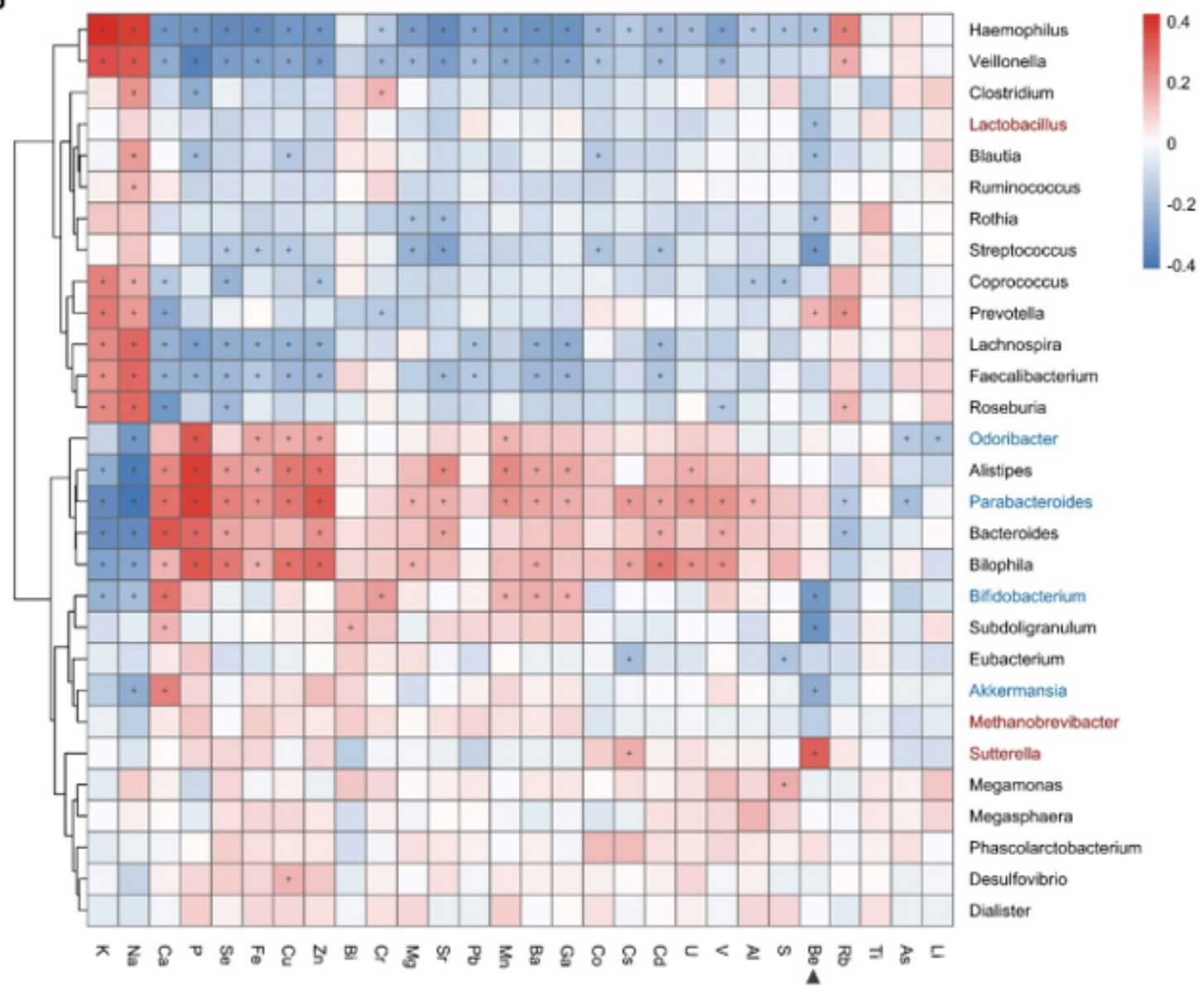

\section{Figure 1}

Associations between fecal elements with MetS risk and human gut microbiota. a Spearman's rank correlation between fecal elements and MetS-related clinical biomarkers. Fecal elements measured from human stools $(n=304)$ are arranged in increasing order of correlation with MetS. Asterisks represent significant associations at FDR adjusted $P$ values of $<0.2$. b Spearman's rank correlation between fecal elements and gut microbiota (genus level). The filtered set of taxa excluding low-abundance taxa $(<0.1 \%$ 
of mean relative abundance) was used for correlation analysis. Asterisks represent significant associations at FDR adjusted $P$ values of $<0.01$. The microbiota marked in blue and red denote taxa significantly enriched in the healthy group and MetS group, respectively. in our previous study [14].

a

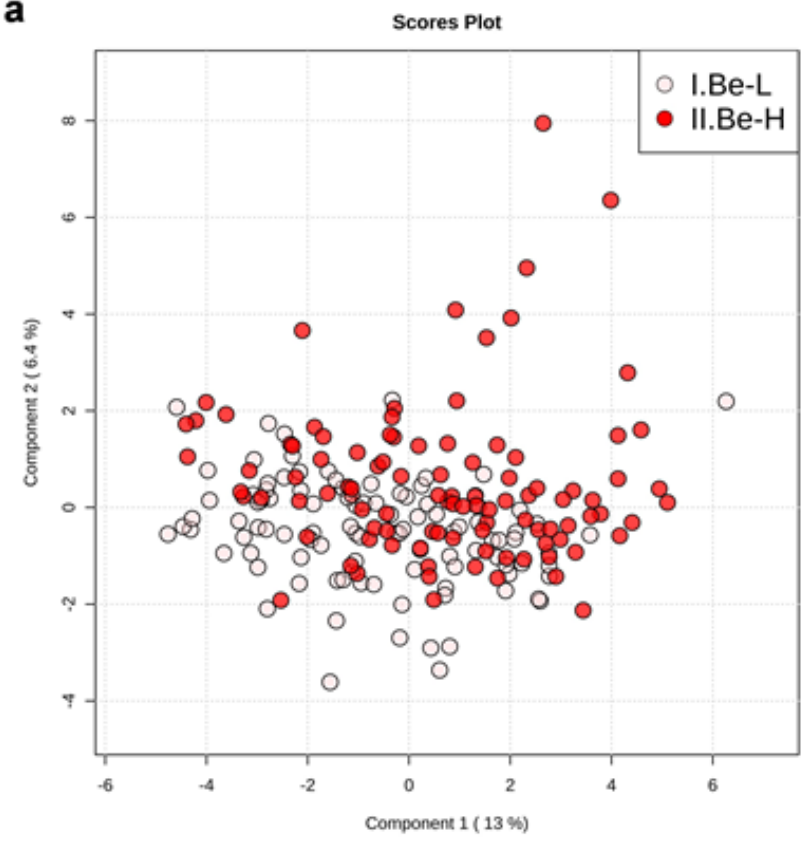

b

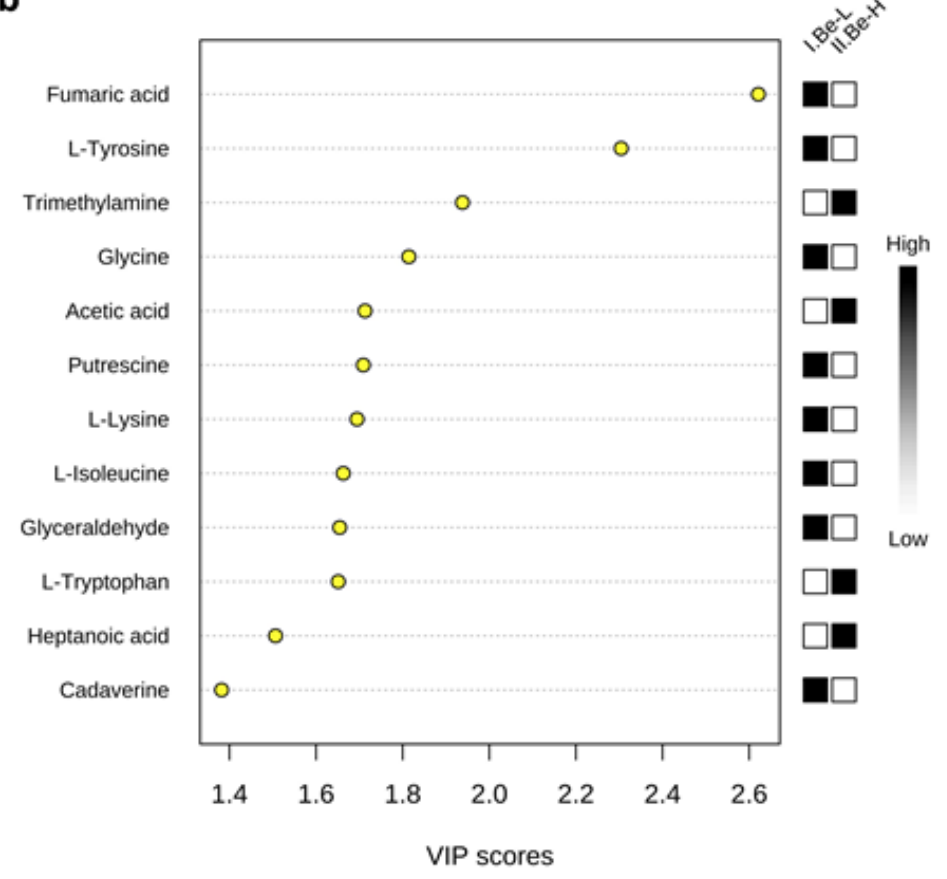

C

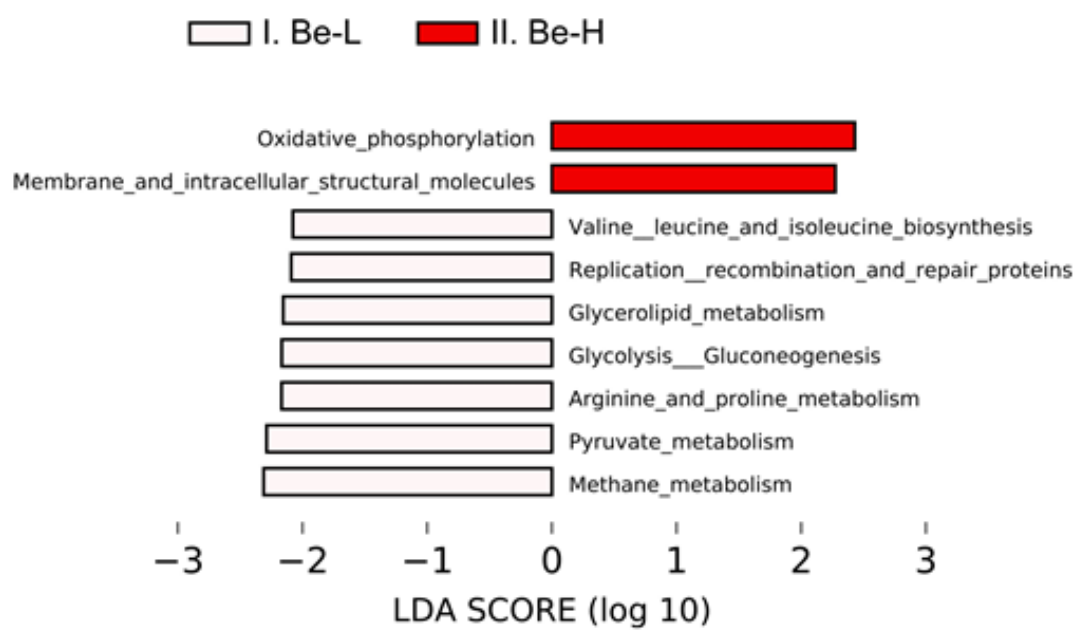

Figure 2

Microbial functional analysis based on fecal Be status. a PLS-DA score plot and $b$ loading plot of NMRbased fecal metabolomic datasets $(n=300)$ based on fecal Be status. The low $(B e-L)$, middle $(B e-M)$, and high $(\mathrm{Be}-\mathrm{H})$ level groups of Be were determined using the tertile values of the relative abundance of elements. Be-L and Be-H groups were compared for the analysis. VIP plots indicate the most discriminating metabolites in descending order of importance. c Histograms of the LDA scores for differentially abundant gut microbial functions according to fecal Be status. Microbial functional profiling was performed using PICRUSt. Features with LDA scores $>2$ presented $(n=304)$. 
a

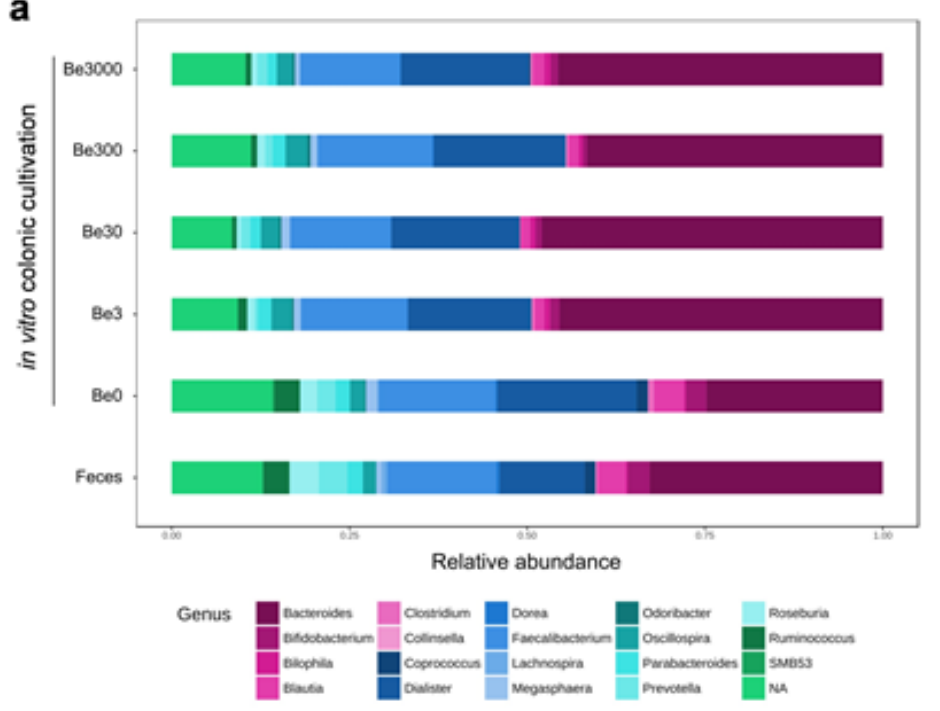

b

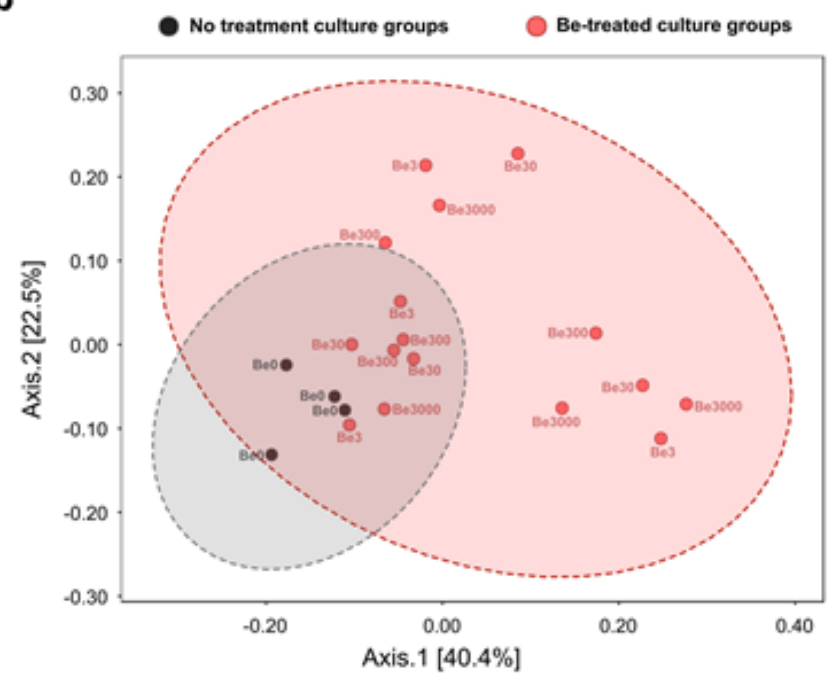

C
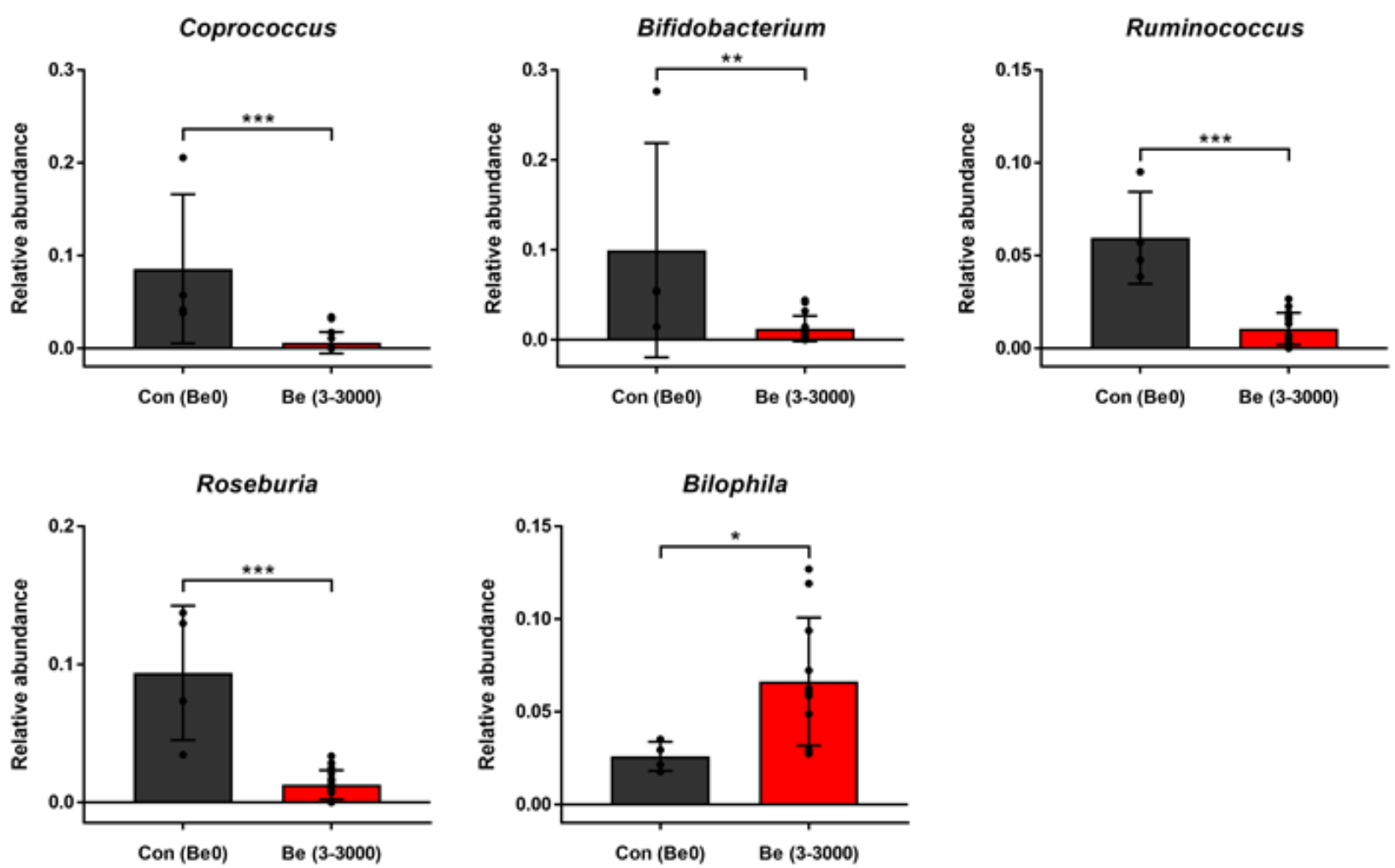

\section{Figure 3}

Perturbation of cultured human fecal microbiota after Be treatment. a Cultured human fecal microbiome composition profiles at the genus level based on the presence of Be. Human feces were collected from three healthy volunteers and cultured under anaerobic conditions with different Be concentrations for 24 h. b PCoA score plot with $95 \%$ confidence ellipse based on unweighted UniFrac metrics was analyzed to investigate the $\beta$-diversity of the community. c Relative abundances for five discriminative taxa, Coprococcus, Bifidobacterium, Ruminococcus, Roseburia, and Bilophila. Data are shown as the mean \pm 
SD. Significance was determined using the Mann-Whitney test vs. control (Be0) group; *P $<0.05$, **P $<$ $0.01, * \star * P<0.001$.
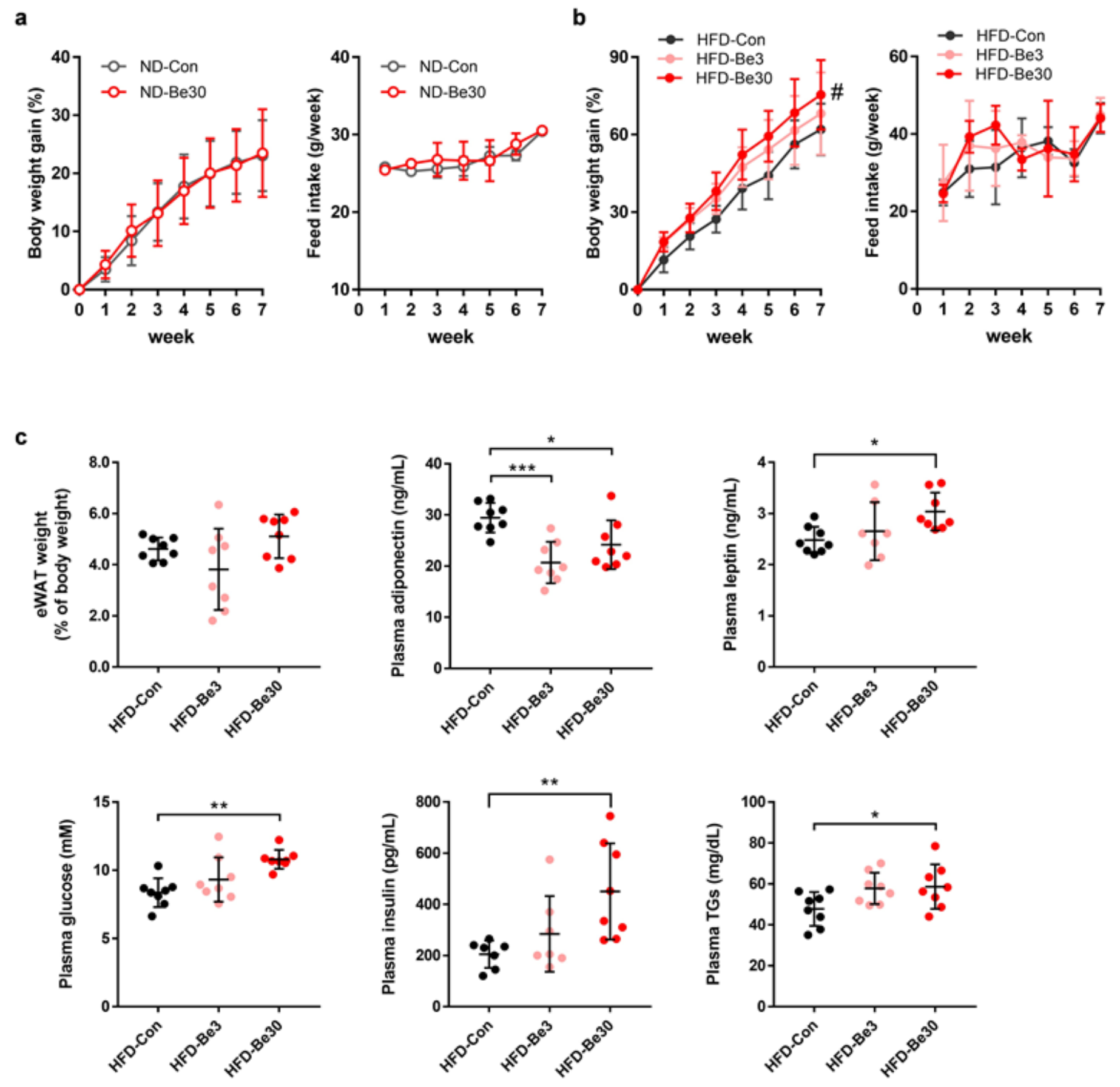

\section{Figure 4}

Effect of Be exposure on mouse host metabolism. Be groups were exposed to Be via drinking water containing 3 ppb or 30 ppb Be for 50 days. a Body weight gain (\%) and feed intake of mice fed a normal $\operatorname{diet}(N D ; n=6 /$ group) and $b$ a high fat diet (HFD; $n=8$ /group). Data represent the means \pm SEM. Significance was determined using two-way ANOVA corrected for multiple comparisons with a Bonferroni test $(\# P<0.05)$ vs. control group. c Epididymal white adipose tissue mass (eWAT), plasma triglycerides 
(TGs), plasma glucose, plasma insulin, plasma leptin, and plasma adiponectin levels in mice fed HFD. Data represent the mean \pm SD. Significance was determined using one-way ANOVA corrected for multiple comparisons with a Sidak test vs. control group; $* \mathrm{P}<0.05$, ${ }^{* *} \mathrm{P}<0.01,{ }^{* \star *} \mathrm{P}<0.001$.
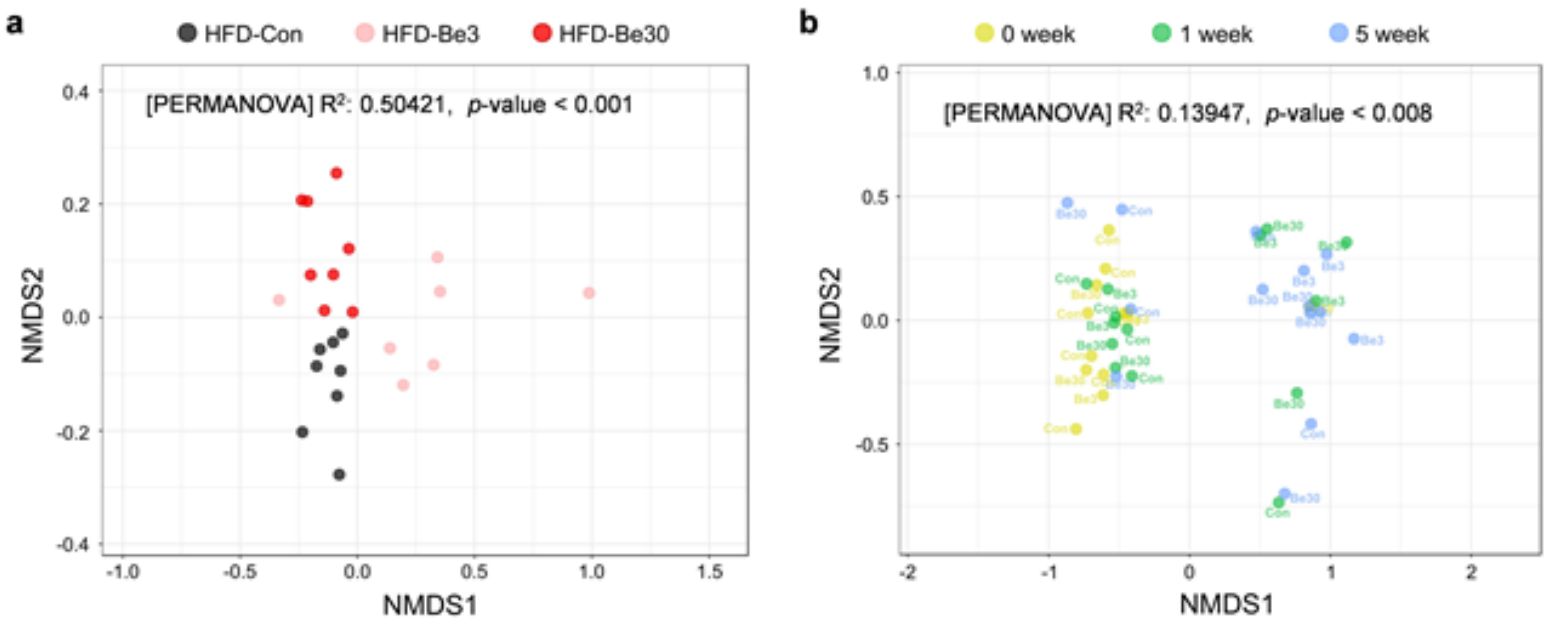

C
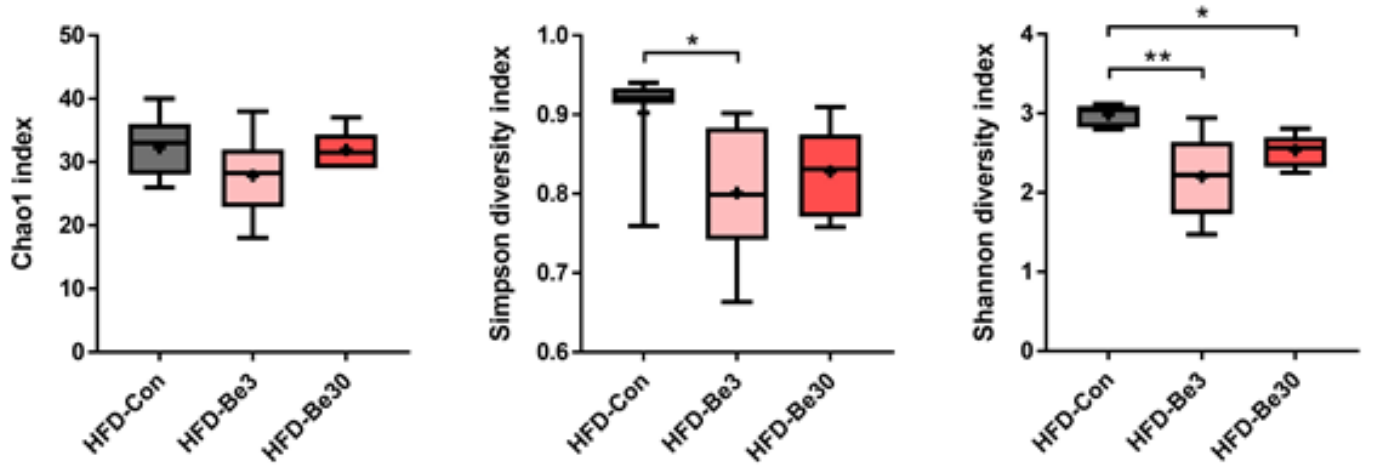

d
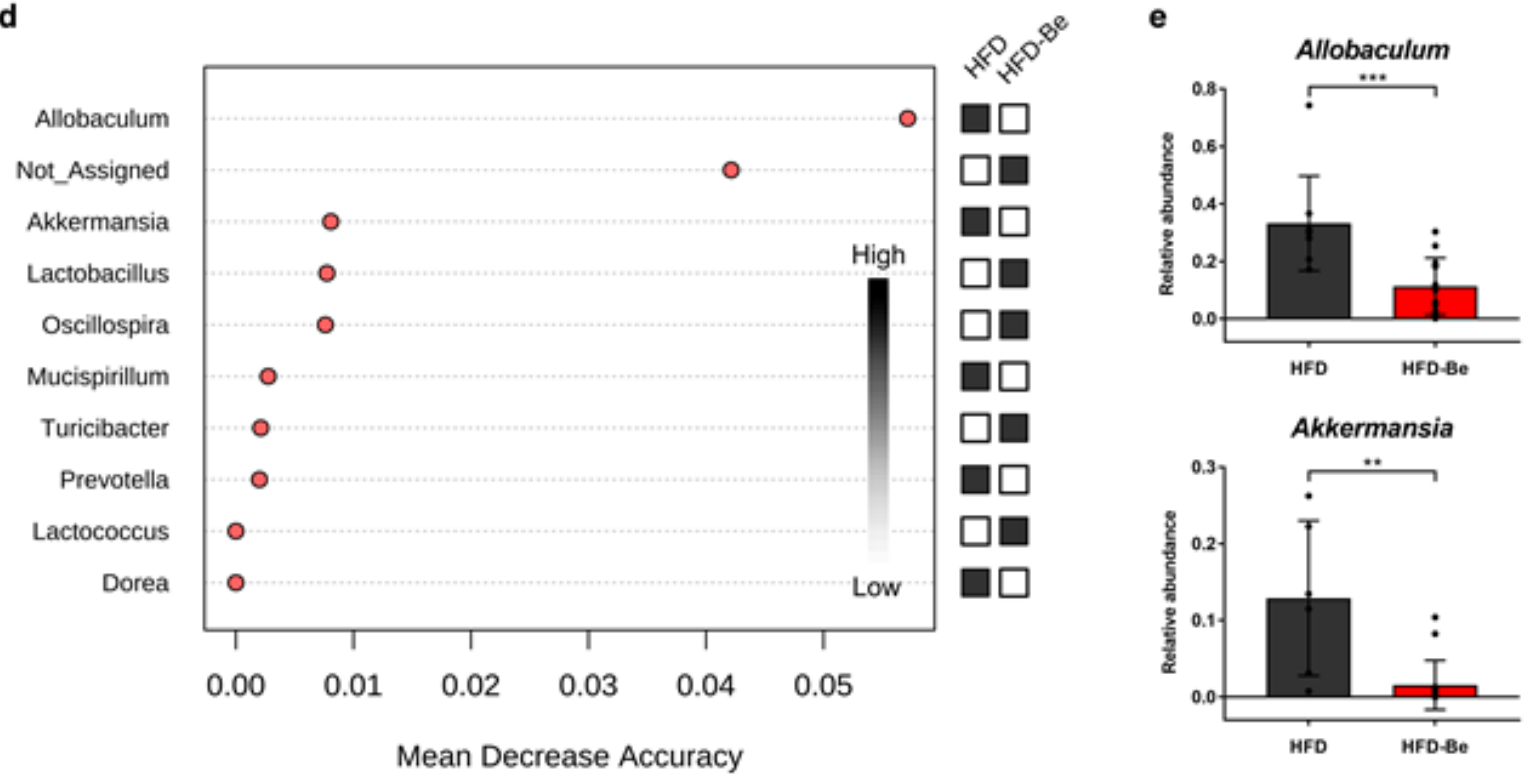

Figure 5

Microbial perturbation in mice fed HFD and exposed to Be. a Non-metric multidimensional scaling (NMDS) plot of the cecal microbiota of Be-exposed HFD-fed mice. b NMDS plot of the fecal microbiota of 
Be exposure over time in HFD-fed mice. Stools were collected at three time points ( 0,1 , and 5 weeks) and analyzed for 16S rRNA gene sequencing. Bray-Curtis distance matrix calculated from the genus-level relative abundance data was used. Significance was determined using PERMANOVA. c Diversity analysis of the cecal microbial community in Be-exposed HFD-fed mice. Chao1 species richness estimator as well as Simpson and Shannon index values for microbial evenness were calculated to investigate the adiversity of each group. Box plots show median (horizontal line), mean (cross), and IQR, while whiskers represent the minimum and maximum values. Significance was determined using unpaired two-tailed Student's t test or one-way ANOVA corrected for multiple comparisons with a Sidak test vs. control group. d Feature importance scores for the ten most predictive genera in the random forest classifier. Feature importance was measured as the mean decrease in model accuracy when that feature's values were permuted randomly. e Relative abundance of two discriminative taxa, Allobaculum and Akkermansia. Data are shown as mean $\pm S D$. Significance was determined using the Mann-Whitney test. ${ }^{*} P<0.05,{ }^{*} P$ $<0.01, * \star * P<0.001$. 
a
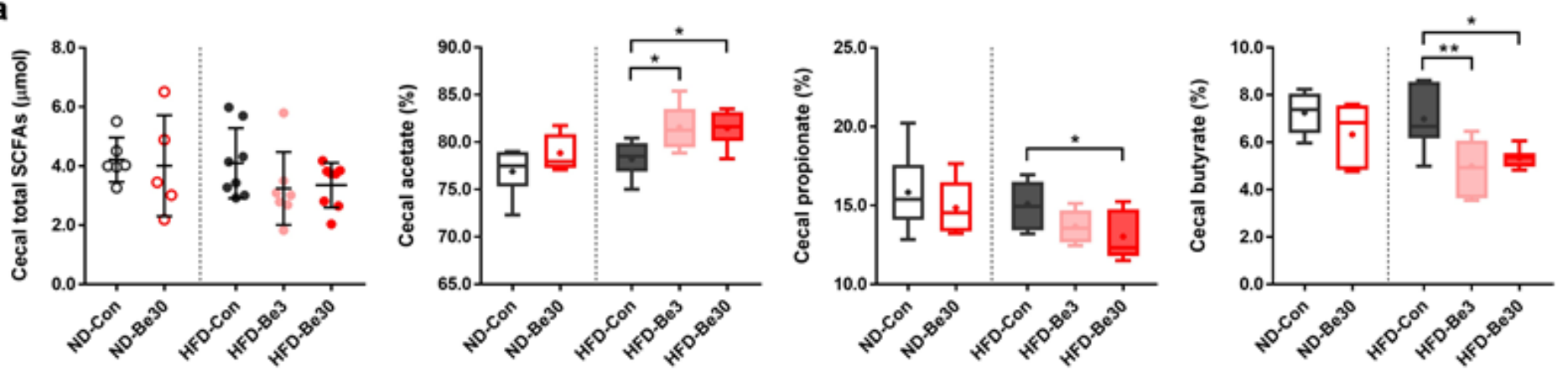

b
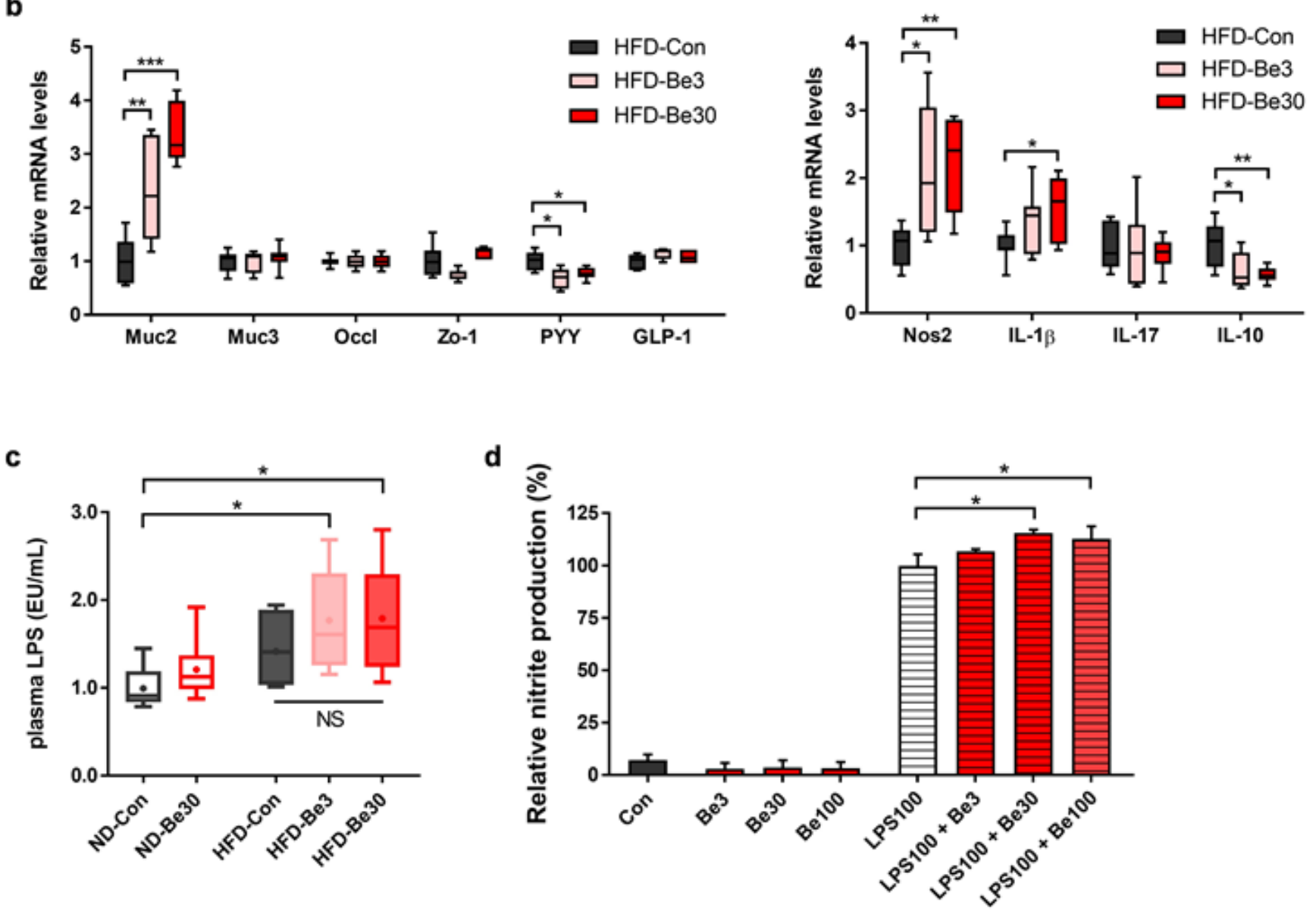

Figure 6

Effect of Be exposure on cecal SCFAs and colonic function. a Cecal SCFA profiles of mice fed an ND or HFD and exposed to Be. b mRNA levels of the mucin production (Muc2 and Muc3), tight junction (Occl and Zo-1), appetite suppression (PYY and GLP-1), and inflammation-related genes in proximal colons of Be-exposed HFD-fed mice. c Plasma LPS levels of mice fed an ND or HFD and exposed to Be. Box plots show median (horizontal line), mean (cross), and IQR, while whiskers represent the minimum and maximum values. Significance was determined using two-tailed Student's $t$ test or one-way ANOVA corrected for multiple comparisons with a Sidak test vs. each control group. $d$ Effect of Be on nitrite production in in vitro cell culture. RAW264.7 cells were treated with different concentrations of Be with or 
without LPS $(100 \mathrm{ng} / \mathrm{mL})$; LPS $(100 \mathrm{ng} / \mathrm{mL})$ was used as a positive control. Data are represented as the mean \pm SEM. Significance was calculated using one-way repeated measures ANOVA corrected for multiple comparisons with a Sidak test vs. positive control. ${ }^{\star} \mathrm{P}<0.05,{ }^{\star \star} \mathrm{P}<0.01$, ${ }^{\star \star \star} \mathrm{P}<0.001$.

\section{Supplementary Files}

This is a list of supplementary files associated with this preprint. Click to download.

- Additionalfile9.docx

- Additionalfile6.tif

- Additionalfile7.tif

- Additionalfile5.tif

- Additionalfile1.tif

- Additionalfile8.tif

- Additionalfile3.tif

- Additionalfile4.docx

- Additionalfile2.docx 